\title{
Modeling the control of atmospheric carbon dioxide through reforestation: effect of time delay
}

\author{
A. K. Misra ${ }^{1}$ - Maitri Verma ${ }^{1} \cdot$ Ezio Venturino $^{2}$
}

Received: 21 July 2015/Accepted: 17 August 2015/Published online: 2 September 2015

(C) Springer International Publishing Switzerland 2015

\begin{abstract}
Carbon dioxide $\left(\mathrm{CO}_{2}\right)$ is the prime greenhouse gas responsible for the threat of global warming. Forest biomass plays an important role in sequestration of carbon dioxide from the atmosphere but the global forest biomass is declining with an alarming rate due to human activities. In this scenario, reforestation is crucial to reduce the atmospheric burden of $\mathrm{CO}_{2}$. In this paper, we propose a nonlinear mathematical model to study the effect of reforestation as well as the delay involved in between the measurement of forest data and implementation of reforestation efforts on the control of atmospheric concentration of $\mathrm{CO}_{2}$. Model analysis shows that the atmospheric concentration of $\mathrm{CO}_{2}$ decreases due to reforestation but a longer delay in between measurement of forest biomass and implementation of reforestation efforts has destabilizing effect on the dynamics of the system. The critical value of this time delay is found analytically. The Hopf-bifurcation analysis is performed by taking time delay as bifurcation parameter. The stability and direction of bifurcating periodic solutions arising through Hopf-bifurcations are also discussed.
\end{abstract}

A. K. Misra

akmisra_knp@yahoo.com; akmisra@bhu.ac.in

Maitri Verma

maitri.verma9@gmail.com

Ezio Venturino

ezio.venturino@unito.it

1 Department of Mathematics, Faculty of Science, Banaras Hindu University, Varanasi 221 005, India

2 Dipartimento di Matematica "Giuseppe Peano", Universita di Torino, via Carlo Alberto 10, 10123 Turin, Italy
Keywords Mathematical model $\cdot \mathrm{CO}_{2}$ gas $\cdot$ Forest biomass - Reforestation efforts - Time delay · Hopf-bifurcation

\section{Introduction}

The enhanced concentration of carbon dioxide $\left(\mathrm{CO}_{2}\right)$ in the Earth's atmosphere is significantly responsible for the menace of global warming and the associated climate changes (IPCC 2007a). The increment in the concentration of atmospheric $\mathrm{CO}_{2}$ is attributed to human activities such as fossil fuel burning and land use changes (IPCC 2007b). Deforestation accounts for nearly $90 \%$ of $\mathrm{CO}_{2}$ emissions from land use changes (IPCC 2001). Forests are one of the major sinks of $\mathrm{CO}_{2}$ on the Earth. Trees during the photosynthesis process absorb $\mathrm{CO}_{2}$ from the atmosphere and stock it in the form of biomass. Every year forests absorb gigatons of carbon in this process. Clearing of forests causes increase in concentration of $\mathrm{CO}_{2}$ in two ways; firstly, it reduces the global uptake of $\mathrm{CO}_{2}$ through photosynthesis process and secondly, the carbon stored in the trees is released back to the atmosphere in the form of $\mathrm{CO}_{2}$ when the wood is burned or left to decompose after deforestation. In the last few decades, global forest biomass has declined at an alarming rate. Between 1990 and 2005, the total forest area has declined at annual rate of $0.21 \%$ and the carbon stock per hectare in forest biomass has declined at the rate $0.02 \%$ (FAO 2010). This severe destruction of forest has contributed significantly to the inexorable rise of atmospheric concentration of $\mathrm{CO}_{2}$.

In this scenario, reforestation is a rational approach to control the atmospheric concentration of $\mathrm{CO}_{2}$ (Woodwell et al. 1983; Goreau 1992). In view of this, reforestation efforts have been made in many countries to increase the 
forest biomass. For this purpose, government estimates the forest biomass and makes efforts for reforestation to maintain the desired level of forest biomass. Since the forests having same area may contain different amount of forest biomass, the estimation of forest biomass is a difficult and time consuming process (Houghton 2005). Consequently, the data of forest biomass used for policy making is usually dated. This time lag involved between measurement of forest biomass and implementation of reforestation efforts may affect the impact of reforestation programs on the control of atmospheric $\mathrm{CO}_{2}$. For designing an effective reforestation policy, it is important to assess the impact of this time lag. In literature, some mathematical models are available which explore the interplay between forest biomass and atmospheric carbon dioxide (Tennakone 1990; Caetano et al. 2011; Misra and Verma 2013; Shukla et al. 2015). In particular, Tennakone (1990) has proposed a mathematical model by considering biomass and $\mathrm{CO}_{2}$ as dynamic variables, finding that excessive deforestation may destabilize the system due to rapid increase in atmospheric $\mathrm{CO}_{2}$. Misra and Verma (2013) have studied the effect of human population and forest biomass on the dynamics of atmospheric $\mathrm{CO}_{2}$, showing that if the deforestation rate exceeds a critical limit, atmospheric concentration of $\mathrm{CO}_{2}$ will not get stabilized. Caetano et al. (2011) have proposed a mathematical model by considering reforestation and clean technology as control variables for the atmospheric $\mathrm{CO}_{2}$ and optimized the total investment on reforestation and clean technology to obtain the desired level of carbon dioxide.

In the present study, we propose and analyze a mathematical model to study the effect of reforestation on the control of atmospheric carbon dioxide by taking measure of reforestation efforts as a dynamical variable. As the reforestation efforts are implemented on the basis of not updated measurements of forest biomass, time delay in implementation of reforestation efforts must be included in the modeling process. The effect of this delay over the dynamics of the system is studied in detail.

\section{Mathematical model}

We assume that the concentration of $\mathrm{CO}_{2}$ in the atmosphere increases due to natural processes as well as human related activities. The emission rate of $\mathrm{CO}_{2}$ from natural sources (e.g., volcanic eruption, respiration process of living organism, changes in oceanic circulation, etc.) is assumed to be a constant. Since the anthropogenic $\mathrm{CO}_{2}$ emissions increase with the expansion in human population (Newel and Marcus 1987; Onozaki 2009); therefore, the anthropogenic emission rate of $\mathrm{CO}_{2}$ is assumed to be proportional to the human population. As forest sequestrates $\mathrm{CO}_{2}$ from the atmosphere during photosynthesis process, we assume that the concentration of $\mathrm{CO}_{2}$ decreases due to increase in forest biomass. The depletion of $\mathrm{CO}_{2}$ due to natural sinks other than forests (like oceans etc.) is assumed to be proportional to atmospheric concentration of $\mathrm{CO}_{2}$ (Nikol'skii 2010). The human population and forest biomass is assumed to follow the logistic growth. Since the climate changes driven by the enhanced concentration of atmospheric $\mathrm{CO}_{2}$ have lethal effects on human life (Casper 2010; McMichael et al. 2006; WHO 2009), therefore we assume that the human population declines due to increase in the concentration of $\mathrm{CO}_{2}$. Man clears forests for food, fuel and other materials as well as land for agriculture and industries, with feedback into the population growth (Brown 1993; Shukla and Dubey 1997; Hartwick 2005; Dubey et al. 2009); thus we assume that the forest biomass decreases due to increase in human population whereas the growth rate of human population is favored by an increase in forest biomass. The reforestation programs are developed on the basis of available data on forest biomass which usually date back in time. Thus we have assumed that the reforestation efforts, which are applied to increase the forest biomass, depend on the difference between the carrying capacity of the forest biomass and its value measured $\tau$ time earlier. Also, we have assumed that some of the reforestation efforts diminish due to their inefficacy or some economical barriers.

Let at any time $t, N(t)$ and $F(t)$ be the human population and forest biomass in any region under consideration. Let $X(t)$ and $R(t)$ be the concentration of $\mathrm{CO}_{2}$ and measure of reforestation efforts, respectively at time $t$. The reforestation efforts can be measured in terms of cost involved in their implementation. In view of the above considerations, the dynamics of the model is governed by the following system of nonlinear delay differential equations:

$$
\begin{aligned}
& \frac{d X}{d t}=Q_{0}+\lambda N-\alpha X-\lambda_{1} X F \\
& \frac{d N}{d t}=s N\left(1-\frac{N}{L}\right)-\theta X N+\pi \phi N F \\
& \frac{d F}{d t}=u F\left(1-\frac{F}{M}\right)-\phi N F+\zeta R F \\
& \frac{d R}{d t}=\gamma(M-F(t-\tau))-\delta_{0} R
\end{aligned}
$$

where $X(0)=X_{0}>0, N(0)=N_{0} \geq 0, F(v)=F_{0} \geq 0$ for $v \in[-\tau, 0], R(0)=R_{0} \geq 0$. The parameters of model system (1) are defined in Table 1.

Here ppm means parts per millions by volume. All the above parameters are assumed to be positive constants. The region of attraction for model system (1) is given by the set: 


$$
\begin{aligned}
\Omega= & \left\{(X, N, F, R) \in \mathbb{R}_{+}^{4}: 0<X \leq X_{m} ; 0 \leq N \leq N_{m} ;\right. \\
& \left.0 \leq F \leq M ; 0 \leq R \leq \frac{\gamma M}{\delta_{0}}\right\}
\end{aligned}
$$

where $X_{m}=\left(Q_{0}+\lambda N_{m}\right) / \alpha, N_{m}=L+\pi \phi L M / s$, and it attracts all the solutions initiating in the interior of the positive orthant.

\section{Equilibrium analysis}

The model system (1) is highly nonlinear, so it is not possible to find its exact solution. Instead, we examine the qualitative behavior of the system using the stability theory of differential equations. We find the equilibrium points of the model and perform the stability analysis of the obtained equilibria. An equilibrium point of a dynamical system is a solution which does not change in time. These points are obtained by putting the growth rate of different variables of model system equal to zero. The model (1) has the following four non-negative equilibria:

1. $E_{1}\left(Q_{0} / \alpha, 0,0, \gamma M / \delta_{0}\right)$ which is always feasible.

2. $E_{2}\left(s\left(Q_{0}+\lambda L\right) /(s \alpha+\theta \lambda L), L\left(s \alpha-\theta Q_{0}\right) /(s \alpha+\theta \lambda L)\right.$, $\left.0, \gamma M / \delta_{0}\right)$ is feasible, provided $s-\frac{\theta Q_{0}}{\alpha}>0$. This condition implies that in absence of forest biomass, the human population thrives only if its intrinsic growth rate is greater than its mortality rate due to $\mathrm{CO}_{2}$.

3. $E_{3}\left(Q_{0} /\left(\alpha+\lambda_{1} M\right), 0, M, 0\right)$ is always feasible.

4. $E_{4}\left(X^{*}, N^{*}, F^{*}, R^{*}\right)$ is feasible provided the following conditions are satisfied:

$$
\begin{aligned}
& u+\frac{\zeta \gamma M}{\delta_{0}}-\phi L\left(\frac{s \alpha-\theta Q_{0}}{s \alpha+\theta \lambda L}\right)>0 \\
& s-\frac{\theta Q_{0}}{\alpha+\lambda_{1} M}+\pi \phi M>0 .
\end{aligned}
$$

The term ' $u+\left(\zeta \gamma M / \delta_{0}\right)-\phi L\left(\left(s \alpha-\theta Q_{0}\right) /(s \alpha+\theta \lambda L)\right)$ ' represents the intrinsic growth rate of the forestry biomass when forest biomass is negligibly small and so it should be positive (otherwise the forest biomass may become extinct). The term ' $s-\left(\theta Q_{0} /\left(\alpha+\lambda_{1} M\right)\right)+\pi \phi M$ ' represents the intrinsic growth rate of the human population when it is negligibly small and hence it should also be positive.

The feasibility of equilibria $E_{1}, E_{2}$ and $E_{3}$ is obvious. The equilibrium $E_{4}\left(X^{*}, N^{*}, F^{*}, R^{*}\right)$ may be obtained by solving the equilibrium equations of (1).

From the fourth equilibrium equation we have

$R=\frac{\gamma(M-F)}{\delta_{0}}$.

Using Eq. (4) in the third equilibrium equation we have
$N=\frac{1}{\phi}\left(u+\frac{\zeta \gamma M}{\delta_{0}}\right)\left(1-\frac{F}{M}\right)$.

From the first equilibrium equation we find

$$
X=\frac{Q_{0}+\lambda N}{\alpha+\lambda_{1} F} .
$$

Using Eqs. (5) and (6) in the second equilibrium equation we obtain the following equation in $F$ :

$$
\begin{aligned}
g(F)= & s\left[1-\frac{1}{\phi L}\left(u+\frac{\zeta \gamma M}{\delta_{0}}\right)\left(1-\frac{F}{M}\right)\right] \\
& -\frac{\theta}{\alpha+\lambda_{1} F}\left[Q_{0}+\frac{\lambda}{\phi}\left(u+\frac{\zeta \gamma M}{\delta_{0}}\right)\left(1-\frac{F}{M}\right)\right]+\pi \phi F .
\end{aligned}
$$

From Eq. (7), we may easily note that:

(1) $g(0)=s\left[1-\frac{1}{\phi L}\left(u+\frac{\zeta \gamma M}{\delta_{0}}\right)\right]-\frac{\theta}{\alpha}\left[Q_{0}+\frac{\lambda}{\phi}\left(u+\frac{\zeta \gamma M}{\delta_{0}}\right)\right]$,

which is negative, if condition (2) holds.

(2) $g(M)=s+\pi \phi M-\frac{\theta Q_{0}}{\alpha+\lambda_{1} M}$,

which is positive, if condition (3) holds.

$$
\begin{aligned}
g^{\prime}(F)= & \frac{s}{\phi L M}\left(u+\frac{\zeta \gamma M}{\delta_{0}}\right)+\frac{\theta}{\left(\alpha+\lambda_{1} F\right)^{2}} \\
& \times\left[\lambda_{1} Q_{0}+\frac{\lambda}{\phi M}\left(u+\frac{\zeta \gamma M}{\delta_{0}}\right)\left(\alpha+\lambda_{1} M\right)\right]+\pi \phi,
\end{aligned}
$$

which is always positive.

Thus a unique positive root $F=F^{*}$ of Eq. (7) exists in the interval $(0, M)$ provided the conditions (2) and (3) are satisfied. Using this value of $F^{*}$ in Eqs. (4), (5) and (6), we get the positive values of $R=R^{*}, N=N^{*}$ and $X=X^{*}$, respectively.

Remark 1 From the equilibrium equations, we can write

$$
\begin{aligned}
& f(X, F, \gamma)=Q_{0}+\frac{\lambda u}{\phi}\left(1-\frac{F}{M}\right)+\frac{\lambda \zeta \gamma}{\phi \delta_{0}}(M-F)-\left(\alpha+\lambda_{1} F\right) X=0, \\
& g(X, F, \gamma)=s-\frac{s u}{L \phi}\left(1-\frac{F}{M}\right)-\frac{s \zeta \gamma}{L \phi \delta_{0}}(M-F)-\theta X+\pi \phi F=0 .
\end{aligned}
$$

From the above equations, we can easily find that

$$
\frac{d X^{*}}{d \gamma}=\frac{-\frac{\zeta}{\delta_{0} \phi}\left(M-F^{*}\right)\left(\frac{\lambda_{1} s}{L} X^{*}-\lambda \pi \phi\right)}{\left(\alpha+\lambda_{1} F^{*}\right)\left(\frac{s u}{L \phi M}+\frac{s \zeta \gamma}{L \delta_{0} \phi}+\pi \phi\right)+\theta\left(\frac{\lambda u}{\phi M}+\frac{\lambda \zeta \gamma}{\delta_{0} \phi}+\lambda_{1} X^{*}\right)} .
$$

Thus, for small value of $\pi, d X^{*} / d \gamma<0$. This implies that if the growth rate of human population due to forest biomass is small, an increase in the implementation rate of reforestation efforts leads to decrease in the atmospheric concentration of $\mathrm{CO}_{2}$. 
Table 1 Model parameters

\begin{tabular}{|c|c|c|}
\hline Parameter & Description & Unit \\
\hline$Q_{0}$ & Emission rate of $\mathrm{CO}_{2}$ from natural sources & ppm year ${ }^{-1}$ \\
\hline$\lambda$ & Emission rate coefficient of $\mathrm{CO}_{2}$ from anthropogenic sources & ppm $(\text { person year) })^{-1}$ \\
\hline$\alpha$ & Natural depletion rate coefficient of atmospheric $\mathrm{CO}_{2}$ & $(\text { year })^{-1}$ \\
\hline$\lambda_{1}$ & Uptake rate coefficient of $\mathrm{CO}_{2}$ by forest biomass & $(\text { ton year })^{-1}$ \\
\hline$s$ & Intrinsic growth rate coefficient of human population & year $^{-1}$ \\
\hline$L$ & Carrying capacity of human population & person \\
\hline$u$ & Intrinsic growth rate coefficient of forest biomass & year $^{-1}$ \\
\hline$M$ & Carrying capacity of forest biomass & ton \\
\hline$\theta$ & Declination rate coefficient of human population due to $\mathrm{CO}_{2}$ & $(\text { ppm year })^{-1}$ \\
\hline$\phi$ & Deforestation rate coefficient & $(\text { person year })^{-1}$ \\
\hline$\pi$ & A proportionality constant which represents growth of human population due to forest biomass & person $(\text { ton })^{-1}$ \\
\hline$\zeta$ & Growth rate coefficient of forest biomass due to reforestation efforts & $(\text { dollar year })^{-1}$ \\
\hline$\gamma$ & Implementation rate coefficient of reforestation efforts & dollar (ton year) $)^{-1}$ \\
\hline$\tau$ & $\begin{array}{l}\text { Time lag involved in between measurement of the data of forest biomass and implementation of reforestation } \\
\text { efforts }\end{array}$ & year \\
\hline$\delta_{0}$ & Declination rate coefficient of reforestation efforts & $(\text { year })^{-1}$ \\
\hline
\end{tabular}

\section{Stability analysis}

In this section, we study the stability behavior of the equilibria with and without delay. We also investigate the possibility of Hopf-bifurcation at the interior equilibrium by taking time delay $\tau$ as a bifurcation parameter. An equilibrium point is stable if either nearby solutions remain nearby for all future time or tend to it. In order to determine the local stability of an equilibrium point, the nature of solutions arbitrarily close to that point is investigated.

\section{Stability analysis without delay (i.e., $\tau=0$ )}

The local stability of an equilibrium point can be determined by finding the sign of the eigenvalues of Jacobian matrix evaluated at that point. The Jacobian matrix for the model system (1) is given by

$$
J=\left(\begin{array}{cccc}
-\left(\alpha+\lambda_{1} F\right) & \lambda & -\lambda_{1} X & 0 \\
-\theta N & s\left(1-\frac{2 N}{L}\right)-\theta X+\pi \phi F & \pi \phi N & 0 \\
0 & -\phi F & u\left(1-\frac{2 F}{M}\right)-\phi N+\zeta R & \zeta F \\
0 & 0 & -\gamma & -\delta_{0}
\end{array}\right) .
$$

Let $J_{i}$ denote the Jacobian matrix $J$ evaluated at equilibrium $E_{i}(i=1,2,3,4)$. By investigating the signs of the eigenvalues of the Jacobian matrices $J_{1}, J_{2}$ and $J_{3}$, we have:

1. The equilibrium $E_{1}$ has a locally stable manifold in $X-R$ plane and an unstable manifold locally in the $F$ direction. Further, $E_{1}$ has a locally unstable manifold in the $N$-direction provided $s-\theta Q_{0} / \alpha>0$. Thus, $E_{1}$ has an unstable manifold locally in the $N$-direction whenever $E_{2}$ is feasible.

2. The equilibrium $E_{2}$ has a stable manifold locally in the $X-N-R$ space. Also, it has an unstable manifold locally in the $F$-direction whenever $E_{4}$ is feasible.

3. The equilibrium $E_{3}$ has a stable manifold locally in the $X-F-R$ space while it has an unstable manifold locally in the $N$-direction whenever $E_{4}$ is feasible.

To investigate the local stability behavior of equilibrium $E_{4}$, we use the Routh-Hurwitz criterion. The characteristic equation for the matrix $J_{4}$ is

$\chi^{4}+A_{1} \chi^{3}+A_{2} \chi^{2}+A_{3} \chi+A_{4}=0$

where, 


$$
\begin{aligned}
A_{1}= & \alpha+\lambda_{1} F^{*}+\frac{s N^{*}}{L}+\frac{u F^{*}}{M}+\delta_{0}, \\
A_{2}= & \delta_{0}\left(\alpha+\lambda_{1} F^{*}+\frac{s N^{*}}{L}+\frac{u F^{*}}{M}\right)+\left(\alpha+\lambda_{1} F^{*}\right) \\
& \times\left(\frac{s N^{*}}{L}+\frac{u F^{*}}{M}\right)+\frac{s u N^{*} F^{*}}{L M}+\pi \phi^{2} N^{*} F^{*}+\lambda \theta N^{*}+\gamma \zeta F^{*}, \\
A_{3}= & \delta_{0}\left(\alpha+\lambda_{1} F^{*}\right)\left(\frac{s N^{*}}{L}+\frac{u F^{*}}{M}\right)+\frac{\delta_{0} s u N^{*} F^{*}}{L M}+\pi \delta_{0} \phi^{2} N^{*} F^{*} \\
& +\lambda \theta \delta_{0} N^{*}+\left(\alpha+\lambda_{1} F^{*}\right)\left(\frac{s u N^{*} F^{*}}{L M}+\pi \phi^{2} N^{*} F^{*}\right) \\
& +\frac{u \lambda \theta N^{*} F^{*}}{M}+\lambda_{1} \theta \phi X^{*} N^{*} F^{*}+\gamma \zeta F^{*}\left(\alpha+\lambda_{1} F+\frac{s N^{*}}{L}\right) \\
A_{4}= & \delta_{0}\left(\alpha+\lambda_{1} F^{*}\right)\left(\frac{s u N^{*} F^{*}}{L M}+\pi \phi^{2} N^{*} F^{*}\right)+\frac{u \lambda \theta \delta_{0} N^{*} F^{*}}{M} \\
& +\delta_{0} \lambda_{1} \theta \phi X^{*} N^{*} F^{*}+\gamma \zeta F^{*}\left(\left(\alpha+\lambda_{1} F^{*}\right) \frac{s N^{*}}{L}+\lambda \theta N^{*}\right)
\end{aligned}
$$

Here, it can be easily noted that $A_{1}, A_{2}, A_{3}$ and $A_{4}$ are positive. The Routh-Hurwitz criterion thus reduces to

$A_{3}\left(A_{1} A_{2}-A_{3}\right)-A_{1}^{2} A_{4}>0$,

Now, we have the following result regarding the local stability of equilibrium $E_{4}$ :

Theorem 1 If feasible, the equilibrium $E_{4}$ is locally asymptotically stable provided the condition (9) holds.

The above theorem tells that if the condition (9) holds, then all the solution trajectories starting nearby the equilibrium $E_{4}$ approaches to $E_{4}$ as $t$ tends to infinity.

Next, we determine the global stability of the equilibrium $E_{4}$ in $\Omega$. The equilibrium point $E_{4}$ is globally asymptotically stable in $\Omega$ if it is asymptotic stable for all initial states in $\Omega$. We use the Lyapunov's direct method to determine global asymptotic stability of $E_{4}$. The basic concept of this method is that if the total energy of a system is diminishing continuously, the system will eventually reach to an equilibrium point and remain at that point. In this method a suitable scalar valued function, called Lyapunov function, is constructed and its first order time derivative along the solution trajectory of the system, is evaluated. If the derivative of Lyapunov function decreases along the solution trajectory as time increases, then the energy of system dissipates and the system settles down to the equilibrium point. We have obtained the following result regarding the global stability of $E_{4}$ :

Theorem 2 If feasible, the equilibrium $E_{4}$ is globally asymptotically stable in $\Omega$ provided the following inequality is satisfied: $\lambda_{1}^{2} X_{m}^{2}<4\left(\alpha+\lambda_{1} F^{*}\right) \frac{\lambda \pi u}{\theta M}$.

Proof Consider the following positive definite function:

$$
\begin{aligned}
V= & \frac{1}{2}\left(X-X^{*}\right)^{2}+m_{1}\left(N-N^{*}-N^{*} \ln \frac{N}{N^{*}}\right) \\
& +m_{2}\left(F-F^{*}-F^{*} \ln \frac{F}{F^{*}}\right)+\frac{m_{3}}{2}\left(R-R^{*}\right)^{2},
\end{aligned}
$$

where $m_{1}, m_{2}$ and $m_{3}$ are positive constants to be chosen appropriately. Now differentiating ' $V$ ' with respect to ' $t$ ' along the solution of system (1), we get

$$
\begin{aligned}
\frac{d V}{d t}= & -\left(\alpha+\lambda_{1} F^{*}\right)\left(X-X^{*}\right)^{2}-\frac{m_{1} s}{L}\left(N-N^{*}\right)^{2} \\
& -\frac{m_{2} u}{M}\left(F-F^{*}\right)^{2}-m_{3} \delta_{0}\left(R-R^{*}\right)^{2}+\left(\lambda-m_{1} \theta\right) \\
& \times\left(X-X^{*}\right)\left(N-N^{*}\right)+\left(m_{1} \pi \phi-m_{2} \phi\right)\left(N-N^{*}\right) \\
& \times\left(F-F^{*}\right)-\lambda_{1} X\left(X-X^{*}\right)\left(F-F^{*}\right) \\
& +\left(m_{2} \zeta-m_{3} \gamma\right)\left(F-F^{*}\right)\left(R-R^{*}\right) .
\end{aligned}
$$

Choosing $m_{1}=\frac{\lambda}{\theta}, m_{2}=\pi m_{1}=\frac{\pi \lambda}{\theta}$ and $m_{3}=\frac{\zeta}{\gamma} m_{2}=\frac{\zeta \pi \lambda}{\gamma \theta}$, we get

$$
\begin{aligned}
\frac{d V}{d t}= & -\left(\alpha+\lambda_{1} F^{*}\right)\left(X-X^{*}\right)^{2}-\frac{\lambda s}{\theta L}\left(N-N^{*}\right)^{2}-\frac{\lambda \pi u}{\theta M}\left(F-F^{*}\right)^{2} \\
& -\frac{\zeta \pi \lambda \delta_{0}}{\gamma \theta}\left(R-R^{*}\right)^{2}-\lambda_{1} X\left(X-X^{*}\right)\left(F-F^{*}\right) .
\end{aligned}
$$

Now we note that $d V / d t$ can be made negative definite inside the region of attraction ' $\Omega$ ' provided condition (10) is satisfied.

\section{Local stability analysis with delay (i.e., $\tau \neq 0$ )}

In the following, we analyze the stability of interior equilibrium $E_{4}$ of (1) with delay (i.e., $\tau \neq 0$ ). We also explore the possibility of Hopf-bifurcation at interior equilibrium $E_{4}$ as the delay parameter $\tau$ varies. Hopf-bifurcation is the phenomenon of the local birth or death of periodic orbits (self-oscillations) from an equilibrium point, as a parameter crosses a critical value. First, we linearize model system (1) about $E_{4}\left(X^{*}, N^{*}, F^{*}, R^{*}\right)$ by using the following transformations:

$X=X^{*}+x, N=N^{*}+n, F=F^{*}+f$ and $R=R^{*}+r$, where $x, n, f$ and $r$ are small perturbations around the equilibrium $E_{4}$. The linearized system of (1) about the equilibrium $E_{4}$ is given as follows:

$\frac{d v}{d t}=M_{1} v(t)+M_{2} v(t-\tau)$ 


$$
\begin{aligned}
& \text { where, } \quad v(t)=[x(t), n(t), f(t), r(t)]^{T} \text {, } \\
& \left(\begin{array}{cccc}
-\left(\alpha+\lambda_{1} F^{*}\right) & \lambda & -\lambda_{1} X^{*} & 0 \\
-\theta N^{*} & -\frac{s N^{*}}{L} & \pi \phi N^{*} & 0 \\
0 & -\phi F^{*} & -\frac{u F^{*}}{M} & \zeta F^{*} \\
0 & 0 & 0 & -\delta_{0}
\end{array}\right) \\
& M_{2}=\left(\begin{array}{cccc}
0 & 0 & 0 & 0 \\
0 & 0 & 0 & 0 \\
0 & 0 & 0 & 0 \\
0 & 0 & -\gamma & 0
\end{array}\right) .
\end{aligned}
$$

The characteristic equation for linearized system (14) is obtained as:

$\Psi^{4}+p_{1} \Psi^{3}+p_{2} \Psi^{2}+p_{3} \Psi+p_{4}+\left(q_{1} \Psi^{2}+q_{2} \Psi+q_{3}\right) e^{-\Psi \tau}=0$

where

$$
\begin{aligned}
p_{1}= & \alpha+\lambda_{1} F^{*}+\frac{s N^{*}}{L}+\frac{u F^{*}}{M}+\delta_{0}, \\
p_{2}= & \delta_{0}\left(\alpha+\lambda_{1} F^{*}+\frac{s N^{*}}{L}+\frac{u F^{*}}{M}\right)+\left(\alpha+\lambda_{1} F^{*}\right) \\
& \times\left(\frac{s N^{*}}{L}+\frac{u F^{*}}{M}\right)+\frac{s u N^{*} F^{*}}{L M}+\pi \phi^{2} N^{*} F^{*}+\lambda \theta N^{*}, \\
p_{3}= & \delta_{0}\left(\alpha+\lambda_{1} F^{*}\right)\left(\frac{s N^{*}}{L}+\frac{u F^{*}}{M}\right)+\frac{\delta_{0} s u N^{*} F^{*}}{L M} \\
& +\pi \delta_{0} \phi^{2} N^{*} F^{*}+\lambda \theta \delta_{0} N^{*} \\
& +\left(\alpha+\lambda_{1} F^{*}\right)\left(\frac{s u N^{*} F^{*}}{L M}+\pi \phi^{2} N^{*} F^{*}\right) \\
& +\frac{u \lambda \theta N^{*} F^{*}}{M}+\lambda_{1} \theta \phi X^{*} N^{*} F^{*}, \\
p_{4}= & \delta_{0}\left(\alpha+\lambda_{1} F^{*}\right)\left(\frac{s u N^{*} F^{*}}{L M}+\pi \phi^{2} N^{*} F^{*}\right) \\
& +\frac{u \lambda \theta \delta_{0} F^{*} N^{*}}{M}+\delta_{0} \lambda_{1} \theta \phi X^{*} N^{*} F^{*}, \\
q_{3}= & \gamma \zeta F^{*}\left(\frac{s N^{*}}{L}\left(\alpha+\lambda_{1} F^{*}\right)+\lambda \theta N^{*}\right) . \\
q_{2}= & \gamma \zeta F^{*}\left(\alpha+\lambda_{1} F^{*}+\frac{s N^{*}}{L}\right) \\
& \\
q_{1} & \\
& \\
&
\end{aligned}
$$

Now, in order to show the Hopf-bifurcation, we have to show that Eq. (15) has a pair of purely imaginary roots. For this purpose, we substitute $\Psi=i \omega(\omega>0)$ in Eq. (15) and separate the real and imaginary parts. We get the following transcendental equations:

$\omega^{4}-p_{2} \omega^{2}+p_{4}=-q_{2} \omega \sin (\omega \tau)-\left(q_{3}-q_{1} \omega^{2}\right) \cos (\omega \tau)$, $p_{1} \omega^{3}-p_{3} \omega=q_{2} \omega \cos (\omega \tau)-\left(q_{3}-q_{1} \omega^{2}\right) \sin (\omega \tau)$.

On squaring and adding the above equations and substituting $\omega^{2}=\rho$, we get

$h(\rho)=\rho^{4}+C_{1} \rho^{3}+C_{2} \rho^{2}+C_{3} \rho+C_{4}=0$,

where $C_{1}=-2 p_{2}+p_{1}^{2}, C_{2}=p_{2}^{2}-2 p_{1} p_{3}+2 p_{4}-q_{1}^{2}, C_{3}=$ $-2 p_{2} p_{4}+p_{3}^{2}+2 q_{1} q_{3}-q_{2}^{2}$ and $C_{4}=p_{4}^{2}-q_{3}^{2}$. If all the coefficients $C_{i}^{\prime} s,(i=1,2,3,4)$ in $h(\rho)$ are positive by Descartes' rule of signs the Eq. (18) will not have any positive real root and thus the characteristic equation (15) will not have a pair of purely imaginary roots. Thus all the roots of Eq. (15) will stay in the negative half plane for $\tau>0$ if they were in the negative half plane for $\tau=0$. In summary:

Theorem 3 If all the coefficients in $h(\rho)$ (i.e., $\left.C_{i}^{\prime} s\right)$ are positive, whenever the interior equilibrium $E_{4}$ is feasible, it is asymptotically stable for all delay $\tau>0$, provided it is stable in the absence of delay.

If we assume the contrary, i.e., the values of all $C_{i}^{\prime} s,(i=$ $1,2,3,4)$ in Eq. (18) are not all positive. Then, by Descartes' rule of signs, we have the following conditions in which the Eq. (18) has exactly one positive root:

$$
\begin{aligned}
& C_{1}>0, C_{2}>0, C_{3}>0, C_{4}<0 \\
& C_{1}<0, C_{2}<0, C_{3}<0, C_{4}<0 \\
& C_{1}>0, C_{2}>0, C_{3}<0, C_{4}<0 \\
& C_{1}>0, C_{2}<0, C_{3}<0, C_{4}<0
\end{aligned}
$$

If any of the above conditions holds then Eq. (15) has only one pair of purely imaginary roots $\pm i \omega_{0}$.

Now, from the transcendental equations (16) and (17), we have

$$
\tan (\omega \tau)=\frac{q_{2} \omega\left(\omega^{4}-p_{2} \omega^{2}+p_{4}\right)+\left(q_{3}-q_{1} \omega^{2}\right)\left(p_{1} \omega^{3}-p_{3} \omega\right)}{\left(q_{3}-q_{1} \omega^{2}\right)\left(\omega^{4}-p_{2} \omega^{2}+p_{4}\right)-q_{2} \omega\left(p_{1} \omega^{3}-p_{3} \omega\right)}
$$

Thus, the value of $\tau_{k}$ corresponding to the positive value $\omega_{0}$ may be obtained as follows:

$$
\begin{aligned}
\tau_{k}= & \frac{k \pi}{\omega_{0}}+\frac{1}{\omega_{0}} \tan ^{-1} \\
& \times\left[\frac{q_{2} \omega_{0}\left(\omega_{0}^{4}-p_{2} \omega_{0}^{2}+p_{4}\right)+\left(q_{3}-q_{1} \omega_{0}^{2}\right)\left(p_{1} \omega_{0}^{3}-p_{3} \omega_{0}\right)}{\left(q_{3}-q_{1} \omega_{0}^{2}\right)\left(\omega_{0}^{4}-p_{2} \omega_{0}^{2}+p_{4}\right)-q_{2} \omega_{0}\left(p_{1} \omega_{0}^{3}-p_{3} \omega_{0}\right)}\right],
\end{aligned}
$$

for $k=0,1,2,3, \ldots$

By using Butler's lemma, we can say that the stable interior equilibrium $E_{4}$ remains stable for $\tau<\tau_{0}$ (Freedman and Rao 1983). We have found the critical value of time delay $\tau_{0}$ which corresponds to the purely imaginary root 
$i \omega_{0}$ of characteristic equation (15). Now we investigate whether or not the phenomenon of Hopf-bifurcation occurs as $\tau$ increases through $\tau_{0}$. For this we need the following lemma.

Lemma 1 The following transversality condition is satisfied: the system (1) undergoes a supercritical Hopf-bifurcation at $\tau=\tau_{0}$, yielding a family of periodic solutions bifurcating from $E_{4}$ as $\tau$ passes through the critical value $\tau_{0}$ (Gopalsamy 1992).

Remark 2 If none of the conditions $(A i)(i=1,2,3,4)$ holds, then Eq. (18) may have more than one positive root. Consequently, there may be more than one pair of purely

$\frac{d \Psi}{d \tau}=\frac{\Psi\left(q_{1} \Psi^{2}+q_{2} \Psi+q_{3}\right) e^{-\Psi \tau}}{4 \Psi^{3}+3 p_{1} \Psi^{2}+2 p_{2} \Psi+p_{3}+\left(2 q_{1} \Psi+q_{2}\right) e^{-\Psi \tau}-\tau\left(q_{1} \Psi^{2}+q_{2} \Psi+q_{3}\right) e^{-\Psi \tau}}$

$\operatorname{sgn}\left[\frac{d(\operatorname{Re}(\Psi))}{d \tau}\right]_{\tau=\tau_{0}}>0$.

Proof Differentiating (15) with respect to $\tau$, we get This gives

$$
\left(\frac{d \Psi}{d \tau}\right)^{-1}=\frac{4 \Psi^{3}+3 p_{1} \Psi^{2}+2 p_{2} \Psi+p_{3}+\left(2 q_{1} \Psi+q_{2}\right) e^{-\Psi \tau}}{\Psi\left(q_{1} \Psi^{2}+q_{2} \Psi+q_{3}\right) e^{-\Psi \tau}}-\frac{\tau}{\Psi}
$$

Now,

$$
\begin{aligned}
\operatorname{sgn}\left[\frac{d(\operatorname{Re}(\Psi))}{d \tau}\right]_{\tau=\tau_{0}} & =\operatorname{sgn}\left[\frac{d(\operatorname{Re}(\Psi))}{d \tau}\right]_{\tau=\tau_{0}}^{-1} \\
& =\operatorname{sgn}\left[\operatorname{Re}\left(\frac{d \Psi}{d \tau}\right)^{-1}\right]_{\Psi=i \omega_{0}} \\
& =\operatorname{sgn}\left[\frac{4 \omega_{0}^{6}+3 C_{1} \omega_{0}^{4}+2 C_{2} \omega_{0}^{2}+C_{3}}{q_{2}^{2} \omega_{0}^{2}+\left(q_{3}-q_{1} \omega_{0}^{2}\right)^{2}}\right] \\
& =\operatorname{sgn}\left[\frac{h^{\prime}\left(\omega_{0}^{2}\right)}{q_{2}^{2} \omega_{0}^{2}+\left(q_{3}-q_{1} \omega_{0}^{2}\right)^{2}}\right] .
\end{aligned}
$$

Here, it may be noted that $h^{\prime}\left(\omega_{0}^{2}\right)>0$ if any one of the conditions $(A i)(i=1,2,3,4)$ is satisfied. This proves the Lemma 1.

Thus the transversality condition holds and this confirms that Hopf-bifurcation occurs at $\tau=\tau_{0}$. Now we have the following result:

Theorem 4 If the condition (9) is satisfied and any one of the condition $(A i)(i=1,2,3,4)$ holds, then the interior equilibrium $E_{4}$ of model (1) is locally asymptotically stable for $\tau \in\left[0, \tau_{0}\right)$ and becomes unstable for $\tau>\tau_{0}$. Further, imaginary roots of Eq. (15) and the system may posses a finite number of stability switches as the delay parameter $\tau$ increases.

\section{Stability and direction of Hopf-bifurcation}

In the previous section, we have obtained the conditions under which the system (1) undergoes a Hopf-bifurcation from the interior equilibrium $E_{4}$ at the critical value of $\tau$. In this section, we investigate the properties of the bifurcating periodic solutions arising through Hopf-bifurcation. Following the idea of Hassard et al., we derive explicit formulae for determining the direction, stability and period of the bifurcating periodic solutions by using the normal form and center manifold theory (Hassard et al. 1981). Without loss of generality, we denote any of the critical values of $\tau$ by $\tau_{k}$ at which Eq. (15) has a pair of purely imaginary roots $\pm i \omega_{0}$ and system (1) undergoes Hopf-bifurcation. Let $\tau=\tau_{k}+\mu, \mu \in \mathbb{R}$, so that $\mu=0$ is a Hopf bifurcation value for the system. Define the space of continuous real valued functions $C=C\left([-1,0], \mathbb{R}^{4}\right)$. Using the transformation $u_{1}(t)=X(t)-X^{*}, u_{2}(t)=N(t)-N^{*}, u_{3}(t)=F(t)-F^{*}$, and $u_{4}(t)=R(t)-R^{*}$ and $x_{i}(t)=u_{i}(\tau t)$ for $i=1,2,3,4$; the delay system (1) transforms into the following functional differential equation in $C$

$\frac{d x}{d t}=L_{\mu} x_{t}+f\left(\mu, x_{t}\right)$

where $\quad x(t)=\left(x_{1}(t), x_{2}(t), x_{3}(t), x_{4}(t)\right)^{T} \in \mathbb{R}^{4}, \quad x_{t}(\Theta)=$ $x(t+\Theta), \Theta \in[-1,0]$ and $L_{\mu}: C \rightarrow \mathbb{R}^{4}, f: C \times \mathbb{R} \rightarrow \mathbb{R}^{4}$ are defined respectively as

$$
L_{\mu} \varphi=\left(\tau_{k}+\mu\right)\left[M_{1} \varphi(0)+M_{2} \varphi(-1)\right],
$$


$f(\mu, \varphi)=\left(\tau_{k}+\mu\right)\left(\begin{array}{c}-\lambda_{1} \varphi_{1}(0) \varphi_{3}(0) \\ -\frac{s}{L} \varphi_{2}^{2}(0)-\theta \varphi_{1}(0) \varphi_{2}(0)+\pi \phi \varphi_{2}(0) \varphi_{3}(0) \\ -\frac{u}{M} \varphi_{3}^{2}(0)-\phi \varphi_{2}(0) \varphi_{3}(0)+\zeta \varphi_{3}(0) \varphi_{4}(0) \\ 0\end{array}\right)$,

with $\varphi=\left(\varphi_{1}, \varphi_{2}, \varphi_{3}, \varphi_{4}\right)^{T} \in C$, and the matrices $M_{1}$ and $M_{2}$ are same as given in previous section.

By the Riesz representation theorem, there exists a function $\eta(\Theta, \mu)$ whose components are of bounded variation for $\Theta \in[-1,0]$ such that

$L_{\mu} \varphi=\int_{-1}^{0} d \eta(\Theta, \mu) \varphi(\Theta)$.

In view of Eq. (26) we can choose

$\eta(\Theta, \mu)=\left(\tau_{k}+\mu\right)\left[M_{1} \delta(\Theta)-M_{2} \delta(\Theta+1)\right]$,

where $\delta(\Theta)$ is the Dirac delta function. For $\varphi \in C^{1}\left([-1,0], \mathbb{R}^{4}\right)$, define

$A(\mu) \varphi=\left\{\begin{array}{lc}\frac{d \varphi(\Theta)}{d \Theta}, & \Theta \in[-1,0), \\ \int_{-1}^{0} d \eta(p, \mu) \varphi(p) \equiv L_{\mu} \varphi, & \Theta=0,\end{array}\right.$

$R(\mu) \varphi=\left\{\begin{array}{lc}0, & \Theta \in[-1,0), \\ f(\varphi, \mu), & \Theta=0 .\end{array}\right.$

Then, the system (25) is equivalent to

$\dot{x}_{t}=A(\mu) x_{t}+R(\mu) x_{t}$,

where $\quad x_{t}(\Theta)=x(t+\Theta)$ for $\Theta \in[-1,0]$. For $\psi \in C^{1}\left([0,1],\left(\mathbb{R}^{4}\right)^{*}\right)$, define

$A^{*} \psi(p)= \begin{cases}-\frac{d \psi(p)}{d p}, & p \in(0,1], \\ \int_{-1}^{0} d \eta^{T}(t, 0) \psi(-t), & p=0 .\end{cases}$

and a bilinear product

$$
\langle\psi, \varphi\rangle=\bar{\psi}(0) . \varphi(0)-\int_{\Theta=-1}^{0} \int_{\xi=0}^{\Theta} \bar{\psi}^{T}(\xi-\Theta) d \eta(\Theta) \varphi(\xi) d \xi,
$$

where $\eta(\Theta)=\eta(\Theta, 0)$ and $\eta^{T}$ denotes the transpose of $\eta$. For convenience, we shall write $A$ and $A^{*}$ in place of $A(0)$ and $A^{*}(0)$ respectively. Then $A$ and $A^{*}$ are adjoint operators. Since $\pm i \omega_{0} \tau_{k}$ are the eigenvalues of $A$, they are also the eigenvalues of $A^{*}$. Now, we need to compute eigenvectors of $A$ and $A^{*}$ corresponding to $+i \omega_{0} \tau_{k}$ and $-i \omega_{0} \tau_{k}$, respectively. Suppose $q(\Theta)=\left(1, \alpha_{1}, \beta_{1}, \gamma_{1}\right)^{T} e^{i \omega_{0} \tau_{k} \Theta}$ be the eigenvector of $A$ corresponding to eigenvalue $i \omega_{0} \tau_{k}$ then

$A q(\Theta)=i \omega_{0} \tau_{k} q(\Theta)$,

for $\Theta=0$, this gives

$$
\begin{gathered}
\left(\begin{array}{cccc}
\alpha+\lambda_{1} F^{*}+i \omega_{0} & -\lambda & \lambda_{1} X^{*} & 0 \\
\theta N^{*} & \frac{s N^{*}}{L}+i \omega_{0} & -\pi \phi N^{*} & 0 \\
0 & \phi F^{*} & \frac{u F^{*}}{M}+i \omega_{0} & -\zeta F^{*} \\
0 & 0 & \gamma e^{-i \omega_{0} \tau_{k}} & \delta_{0}+i \omega_{0}
\end{array}\right) \\
\left(\begin{array}{l}
1 \\
\alpha_{1} \\
\beta_{1} \\
\gamma_{1}
\end{array}\right)=\left(\begin{array}{l}
0 \\
0 \\
0 \\
0
\end{array}\right) .
\end{gathered}
$$

Solving the system of Eq. (36), we get

$$
\begin{aligned}
& \alpha_{1}=\frac{\pi \phi N^{*}\left(\alpha+\lambda_{1} F^{*}+i \omega_{0}\right)+\lambda_{1} \theta X^{*} N^{*}}{\pi \phi \lambda N^{*}-\lambda_{1} X^{*}\left(\frac{s N^{*}}{L}+i \omega_{0}\right)}, \\
& \beta_{1}=\frac{\lambda_{1} X^{*}\left(\frac{s N^{*}}{L}+i \omega_{0}\right)\left(\alpha+\lambda_{1} F^{*}+i \omega_{0}\right)+\lambda \lambda_{1} \theta X^{*} N^{*}}{\lambda_{1} X^{*}\left(\pi \phi \lambda N^{*}-\lambda_{1} X^{*}\left(\frac{s N^{*}}{L}+i \omega_{0}\right)\right)} \\
& \text { and } \gamma_{1}=\frac{-\gamma e^{-i \omega_{0} \tau_{k}} \beta_{1}}{\delta_{0}+i \omega_{0}} .
\end{aligned}
$$

Similarly, we calculate $q^{*}(p)=D\left(1, \alpha_{1}^{*}, \beta_{1}^{*}, \gamma_{1}^{*}\right)^{T} e^{i \omega_{0} \tau_{k} p}$ such that

$A^{*} q^{*}(p)=-i \omega_{0} \tau_{k} q^{*}(p)$

where $\alpha_{1}^{*}=\frac{-\left(\alpha+\lambda_{1} F^{*}\right)+i \omega_{0}}{F^{*} \beta^{*}}, \beta_{1}^{*}=\frac{\lambda+\left(-\frac{s N^{*}}{L}+i \omega_{0}\right) \alpha_{1}^{*}}{\phi F^{*}}$ and $\gamma_{1}^{*}=\frac{\zeta F^{*} \beta_{1}^{*}}{\delta_{0}-i \omega_{0}}$.

Now, we need to determine the value of $\mathrm{D}$ such that $\left\langle q^{*}(p), q(\Theta)\right\rangle=1$.

Using (34), we have

$$
\begin{aligned}
& \overline{q^{*}}(0) \cdot q(0)-\int_{\Theta=-1}^{0} \int_{\xi=0}^{\Theta} \overline{q^{*}}(0) e^{-i \omega_{0} \tau_{k}(\xi-\Theta)} d \eta(\Theta) q(0) e^{i \omega_{0} \tau_{k} \xi} d \xi \\
& \bar{D}\left[1+\alpha_{1} \overline{\alpha_{1}^{*}}+\beta_{1} \overline{\beta_{1}^{*}}+\gamma_{1} \overline{\gamma_{1}^{*}}-\tau_{k} \gamma \beta_{1} \overline{\gamma_{1}^{*}} e^{-i \omega_{0} \tau_{k}}\right]
\end{aligned}
$$

Thus, $D$ is chosen such that

$\bar{D}=\frac{1}{1+\alpha_{1} \overline{\alpha_{1}^{*}}+\beta_{1} \overline{\beta_{1}^{*}}+\gamma_{1} \overline{\gamma_{1}^{*}}-\tau_{k} \gamma \beta_{1} \overline{\gamma_{1}^{*}} e^{-i \omega_{0} \tau_{k}}}$.

Moreover, we can verify that $\left\langle q^{*}(p), \overline{q(\Theta)}\right\rangle=0$.

In the following, we proceed as Hassard et al. (1981) to compute the coordinates describing the center manifold $C_{0}$ at $\mu=0$. Let $x_{t}$ be the solution of Eq. (32) when $\mu=0$. Define 
$z(t)=\left\langle q^{*}, x_{t}\right\rangle, W(t, \Theta)=x_{t}(\Theta)-2 \operatorname{Re}\{z(t) q(\Theta)\}$.

On the center manifold $C_{0}$, we have

$W(t, \Theta)=W(z, \bar{z}, \Theta)$,

where

$$
W(z, \bar{z}, \Theta)=W_{20}(\Theta) \frac{z^{2}}{2}+W_{11}(\Theta) z \bar{z}+W_{02}(\Theta) \frac{\bar{z}^{2}}{2}+\cdots
$$

$z$ and $\bar{z}$ are local coordinates for the center manifold $C_{0}$ in the direction of $q^{*}$ and $\bar{q}^{*}$, respectively. Note that $W$ is real if $x_{t}$ is real. We will consider real solutions only. From (39), we have

$$
\left\langle q^{*}, W\right\rangle=\left\langle q^{*}, x_{t}-z q-\overline{z q}\right\rangle=0 .
$$

For a real solution $x_{t} \in C_{0}$ of equation (32), we have

$$
\begin{aligned}
\dot{z} & =i \omega_{0} \tau_{k} z+\overline{q^{*}}(0) \cdot f(0, W(z, \bar{z}, 0)+2 \operatorname{Re}\{z q(0)\}) \\
& =i \omega_{0} \tau_{k} z+\overline{q^{*}}(0) \cdot f_{0}(z, \bar{z}) .
\end{aligned}
$$

This equation can be rewritten as

$$
\dot{z}=i \omega_{0} \tau_{k} z+g(z, \bar{z}),
$$

where

$$
\begin{aligned}
g(z, \bar{z})= & \overline{q^{*}}(0) \cdot f_{0}(z, \bar{z})=g_{20} \frac{z^{2}}{2}+g_{11} z \bar{z} \\
& +g_{02} \frac{\bar{z}^{2}}{2}+g_{21} \frac{z^{2} \bar{z}}{2}+\cdots
\end{aligned}
$$

It follows from (39) and (41) that

$$
\begin{aligned}
x_{t}(\Theta)=W(z, \bar{z}, \Theta)+2 \operatorname{Re}\{z q(\Theta)\} \\
=W_{20}(\Theta) \frac{z^{2}}{2}+W_{11}(\Theta) z \bar{z}+W_{02}(\Theta) \frac{\bar{z}^{2}}{2} \\
\quad+z\left(1, \alpha_{1}, \beta_{1}, \gamma_{1}\right)^{T} e^{i \omega_{0} \tau_{k} \Theta} \\
\quad+\bar{z}\left(1, \overline{\alpha_{1}}, \overline{\beta_{1}}, \overline{\gamma_{1}}\right)^{T} e^{-i \omega_{0} \tau_{k} \Theta}+\cdots
\end{aligned}
$$

so that

$$
\begin{aligned}
x_{1 t}(\Theta)= & W_{20}^{(1)}(\Theta) \frac{z^{2}}{2}+W_{11}^{(1)}(\Theta) z \bar{z}+W_{02}^{(1)}(\Theta) \frac{\bar{z}^{2}}{2} \\
& +z e^{i \omega_{0} \tau_{k} \Theta}+\bar{z} e^{-i \omega_{0} \tau_{k} \Theta}+\cdots \\
x_{2 t}(\Theta)= & W_{20}^{(2)}(\Theta) \frac{z^{2}}{2}+W_{11}^{(2)}(\Theta) z \bar{z}+W_{02}^{(2)}(\Theta) \frac{\bar{z}^{2}}{2} \\
& +\alpha_{1} e^{i \omega_{0} \tau_{k} \Theta} z+\overline{\alpha_{1}} e^{-i \omega_{0} \tau_{k} \Theta} \bar{z}+\cdots \\
x_{3 t}(\Theta)= & W_{20}^{(3)}(\Theta) \frac{z^{2}}{2}+W_{11}^{(3)}(\Theta) z \bar{z}+W_{02}^{(3)}(\Theta) \frac{\bar{z}^{2}}{2} \\
& +\beta_{1} e^{i \omega_{0} \tau_{k} \Theta} z+\overline{\beta_{1}} e^{-i \omega_{0} \tau_{k} \Theta} \bar{z}+\cdots \\
x_{4 t}(\Theta)= & W_{20}^{(4)}(\Theta) \frac{z^{2}}{2}+W_{11}^{(4)}(\Theta) z \bar{z}+W_{02}^{(4)}(\Theta) \frac{\bar{z}^{2}}{2} \\
& +\gamma_{1} e^{i \omega_{0} \tau_{k} \Theta} z+\bar{\gamma}_{1} e^{-i \omega_{0} \tau_{k} \Theta} \bar{z}+\cdots
\end{aligned}
$$

Thus, we have

$$
\begin{aligned}
& x_{1 t}(0)=z+\bar{z}+W_{20}^{(1)}(0) \frac{z^{2}}{2}+W_{11}^{(1)}(0) z \bar{z}+W_{02}^{(1)}(0) \frac{\bar{z}^{2}}{2}+\cdots \\
& x_{2 t}(0)=\alpha_{1} z+\overline{\alpha_{1}} \bar{z}+W_{20}^{(2)}(0) \frac{z^{2}}{2}+W_{11}^{(2)}(0) z \bar{z}+W_{02}^{(2)}(0) \frac{\bar{z}^{2}}{2}+\cdots \\
& x_{3 t}(0)=\beta_{1} z+\overline{\beta_{1}} \bar{z}+W_{20}^{(3)}(0) \frac{z^{2}}{2}+W_{11}^{(3)}(0) z \bar{z}+W_{02}^{(3)}(0) \frac{\bar{z}^{2}}{2}+\cdots \\
& x_{4 t}(0)=\gamma_{1} z+\overline{\gamma_{1}} \bar{z}+W_{20}^{(4)}(0) \frac{z^{2}}{2}+W_{11}^{(4)}(0) z \bar{z}+W_{02}^{(4)}(0) \frac{\bar{z}^{2}}{2}+\cdots
\end{aligned}
$$

From the definition of $f(\mu, \varphi)$, we have

$$
\begin{aligned}
g(z, \bar{z})= & \overline{q^{*}}(0) \cdot f\left(0, x_{t}\right)=\tau_{k} \bar{D}\left(1, \overline{\alpha_{1}^{*}}, \overline{\beta_{1}^{*}}, \overline{\gamma_{1}^{*}}\right)^{T} . \\
& \left(\begin{array}{c}
-\lambda_{1} x_{1 t}(0) x_{3 t}(0) \\
-\frac{s}{L} x_{2 t}^{2}(0)-\theta x_{1 t}(0) x_{2 t}(0)+\pi \phi x_{2 t}(0) x_{3 t}(0) \\
-\frac{u}{M} x_{3 t}^{2}(0)-\phi x_{2 t}(0) x_{3 t}(0)+\zeta x_{3 t}(0) x_{4 t}(0) \\
0
\end{array}\right) .
\end{aligned}
$$

Using the expressions for $x_{1 t}(0), x_{2 t}(0), x_{3 t}(0)$ and $x_{4 t}(0)$ from (47) in (48) and comparing the coefficients of $z^{2}, z \bar{z}$, $\bar{z}^{2}$ and $z^{2} \bar{z}$ of the resulting expression with those in (44), we get

$$
\begin{aligned}
g_{20}= & 2 \tau_{k} \bar{D}\left[-\lambda_{1} \beta_{1}-\frac{s}{L} \alpha_{1}^{2} \overline{\alpha_{1}^{*}}-\theta \alpha_{1} \overline{\alpha_{1}^{*}}+\pi \phi \alpha_{1} \beta_{1} \overline{\alpha_{1}^{*}}\right. \\
& \left.-\frac{u}{M} \beta_{1}^{2} \overline{\beta_{1}^{*}}-\phi \alpha_{1} \beta_{1} \overline{\beta_{1}^{*}}+\zeta \beta_{1} \bar{\gamma}_{1} \overline{\beta_{1}^{*}}\right] \\
g_{11}= & 2 \tau_{k} \bar{D}\left[-\lambda_{1} \operatorname{Re}\left\{\beta_{1}\right\}-\frac{s}{L}\left|\alpha_{1}\right|^{2} \overline{\alpha_{1}^{*}}-\theta \operatorname{Re}\left\{\alpha_{1}\right\} \overline{\alpha_{1}^{*}}\right. \\
& +\pi \phi \operatorname{Re}\left\{\overline{\alpha_{1}} \overline{\beta_{1}}\right\} \overline{\alpha_{1}^{*}}-\frac{u}{M}\left|\beta_{1}\right|^{2} \overline{\beta_{1}^{*}}-\phi \operatorname{Re}\left\{\overline{\alpha_{1}} \overline{\beta_{1}}\right\} \overline{\beta_{1}^{*}} \\
& \left.+\zeta \operatorname{Re}\left\{\beta_{1} \overline{\gamma_{1}}\right\} \overline{\beta_{1}^{*}}\right] \\
g_{02}= & 2 \tau_{k} \bar{D}\left[-\lambda_{1} \overline{\beta_{1}}-\frac{s}{L}{\overline{\alpha_{1}}}^{2} \overline{\alpha_{1}^{*}}-\theta \overline{\alpha_{1}} \overline{\alpha_{1}^{*}}+\pi \phi \overline{\alpha_{1}} \overline{\beta_{1} \overline{\alpha_{1}^{*}}}\right. \\
& \left.-\frac{u}{\bar{M}} \overline{\beta_{1}} \overline{\beta_{1}^{*}}-\phi \overline{\alpha_{1}} \overline{\beta_{1} \bar{\beta}_{1}^{*}}+\zeta \overline{\beta_{1}} \overline{\gamma_{1}} \overline{\beta_{1}^{*}}\right] \\
g_{21}= & \tau_{k} \bar{D}\left[-\lambda_{1}\left(2 W_{11}^{(3)}(0)+W_{20}^{(3)}(0)+\overline{\beta_{1}} W_{20}^{(1)}(0)\right.\right. \\
& \left.+2 \beta_{1} W_{11}^{(1)}(0)\right)-2 \overline{\alpha_{1}^{*}} \frac{s}{L}\left(2 \alpha_{1} W_{11}^{(2)}(0)+\overline{\alpha_{1}} W_{20}^{(2)}(0)\right) \\
& -\theta \overline{\alpha_{1}^{*}}\left(2 W_{11}^{(2)}(0)+W_{20}^{(2)}(0)+\overline{\alpha_{1}} W_{20}^{(1)}(0)\right. \\
& \left.+2 \alpha_{1} W_{11}^{(1)}(0)\right)+\left(\pi \phi \overline{\alpha_{1}^{*}}-\phi \overline{\beta_{1}^{*}}\right)\left(2 \alpha_{1} W_{11}^{(3)}(0)\right. \\
& \left.+\overline{\alpha_{1}} W_{20}^{(3)}(0)+\overline{\beta_{1}} W_{20}^{(2)}(0)+2 \beta_{1} W_{11}^{(2)}(0)\right) \\
& -2 \overline{\beta_{1}^{*}} \frac{u}{M}\left(\overline{\beta_{1}} W_{20}^{(3)}(0)+2 \beta_{1} W_{11}^{(3)}(0)\right)+\zeta \overline{\beta_{1}^{*}}\left(2 \beta_{1} W_{11}^{(4)}(0)\right. \\
& \left.\left.+\overline{\beta_{1}} W_{20}^{(4)}(0)+2 \gamma_{1} W_{11}^{(3)}(0)+\overline{\gamma_{1}} W_{20}^{(3)}(0)\right)\right]
\end{aligned}
$$

In order to compute $g_{21}$, we still need to compute $W_{20}(\Theta)$ and $W_{11}(\Theta)$. From Eqs. (39) and (42), we have 


$$
\begin{aligned}
& \dot{W}=\dot{x_{t}}-\dot{z} q-\dot{\bar{z}} \bar{q} \\
& =\left\{\begin{array}{lc}
A W-2 \operatorname{Re}\left\{\overline{q^{*}}(0) \cdot f_{0} q(\Theta)\right\}, & \Theta \in[-1,0), \\
A W-2 \operatorname{Re}\left\{\overline{q^{*}}(0) \cdot f_{0} q(0)\right\}+f_{0}, & \Theta=0,
\end{array}\right. \\
& \equiv A W+H(z, \bar{z}, \Theta)
\end{aligned}
$$

where

$H(z, \bar{z}, \Theta)=H_{20}(\Theta) \frac{z^{2}}{2}+H_{11}(\Theta) z \bar{z}+H_{02}(\Theta) \frac{\bar{z}^{2}}{2}+\cdots$

Also, on the center manifold $C_{0}$ near the origin,

$$
\dot{W}=W_{z} \dot{z}+W_{\bar{z}} \dot{\bar{z}}
$$

Using Eqs. (41), (43), (50) and (51) in (52) and comparing the coefficients of $z^{2}$ and $z \bar{z}$ on both sides, we obtain

$$
\begin{aligned}
\left(A-2 i \omega_{0} \tau_{k}\right) W_{20} & =-H_{20}, \\
A W_{11} & =-H_{11} .
\end{aligned}
$$

Further, for $\Theta \in[-1,0)$, we have

$$
\begin{aligned}
H(z, \bar{z}, \Theta)= & -\bar{q}^{*}(0) \cdot f_{0} q(\Theta)-q^{*}(0) \cdot \bar{f}_{0} \bar{q}(\Theta) \\
= & -g(z, \bar{z}) q(\Theta)-\bar{g}(z, \bar{z}) \bar{q}(\Theta) \\
= & -\left(g_{20} q(\Theta)+\bar{g}_{02} \bar{q}(\Theta)\right) \frac{z^{2}}{2}-\left(g_{11} q(\Theta)\right. \\
& \left.+\bar{g}_{11} \bar{q}(\Theta)\right) z \bar{z}+\cdots,
\end{aligned}
$$

which on comparing the coefficients with (51) gives

$H_{20}(\Theta)=-g_{20} q(\Theta)-\bar{g}_{02} \bar{q}(\Theta)$

and

$H_{11}(\Theta)=-g_{11} q(\Theta)-\bar{g}_{11} \bar{q}(\Theta)$.

From (53), (55) and the definition of $A$, we have

$W_{20}^{\prime}(\Theta)=2 i \omega_{0} \tau_{k} W_{20}(\Theta)+g_{20} q(\Theta)+\bar{g}_{02} \bar{q}(\Theta)$.

Note that $q(\Theta)=q(0) e^{i \omega_{0} \tau_{k} \Theta}$, hence

$W_{20}(\Theta)=\frac{i g_{20}}{\omega_{0} \tau_{k}} q(\Theta)+\frac{i \bar{g}_{02}}{3 \omega_{0} \tau_{k}} \bar{q}(\Theta)+F_{1} e^{2 i \omega_{0} \tau_{k} \Theta}$.

Similarly from (53), (56) and the definition of $A$, we have

$W_{11}^{\prime}(\Theta)=g_{11} q(\Theta)+\bar{g}_{11} \bar{q}(\Theta)$,

which gives

$W_{11}(\Theta)=-\frac{i g_{11}}{\omega_{0} \tau_{k}} q(\Theta)+\frac{i \bar{g}_{11}}{\omega_{0} \tau_{k}} \bar{q}(\Theta)+F_{2}$.

where $\quad F_{1}=\left(F_{1}^{(1)}, F_{1}^{(2)}, F_{1}^{(3)}, F_{1}^{(4)}\right) \quad$ and $\quad F_{2}=$ $\left(F_{2}^{(1)}, F_{2}^{(2)}, F_{2}^{(3)}, F_{2}^{(3)}\right) \in \mathbb{R}^{4}$ are constant vectors, to be determined. It follows from the definition of $A$ and (53) that $\int_{-1}^{0} d \eta(\Theta) W_{20}(\Theta)=2 i \omega_{0} \tau_{k} W_{20}(0)-H_{20}(0)$

$\int_{-1}^{0} d \eta(\Theta) W_{11}(\Theta)=-H_{11}(0)$.

From Eqs. (49) and (51), we get

$H_{20}(0)=-g_{20} q(0)-\bar{g}_{02} \bar{q}(0)+2 \tau_{k}\left(\begin{array}{c}-\lambda_{1} \beta_{1} \\ -\frac{s}{L} \alpha_{1}^{2}-\theta \alpha_{1}+\pi \phi \alpha_{1} \beta_{1} \\ -\frac{u}{M} \beta_{1}^{2}-\phi \alpha_{1} \beta_{1}+\zeta \beta_{1} \gamma_{1} \\ 0\end{array}\right)$

and

$$
\begin{aligned}
& H_{11}(0)=-g_{11} q(0)-\bar{g}_{11} \bar{q}(0) \\
&+2 \tau_{k}\left(\begin{array}{c}
-\lambda_{1} \operatorname{Re}\left\{\beta_{1}\right\} \\
-\frac{s}{L}\left|\alpha_{1}\right|^{2}-\theta \operatorname{Re}\left\{\alpha_{1}\right\}+\pi \phi \operatorname{Re}\left\{\alpha_{1} \overline{\beta_{1}}\right\} \\
-\frac{u}{M}\left|\beta_{1}\right|^{2}-\phi \operatorname{Re}\left\{\alpha_{1} \overline{\beta_{1}}\right\}+\zeta \operatorname{Re}\left\{\beta_{1} \overline{\gamma_{1}}\right\} \\
0
\end{array}\right) .
\end{aligned}
$$

Using (58) and (63) in (61) and noting that $q(\Theta)$ is eigenvector of $A$, we have

$$
\begin{aligned}
& \left(2 i \omega_{0} \tau_{k} I-\int_{-1}^{0} e^{2 i \omega_{0} \tau_{k} \Theta} d \eta(\Theta)\right) F_{1} \\
& =2 \tau_{k}\left(\begin{array}{c}
-\lambda_{1} \beta_{1} \\
-\frac{s}{L} \alpha_{1}^{2}-\theta \alpha_{1}+\pi \phi \alpha_{1} \beta_{1} \\
-\frac{u}{M} \beta_{1}^{2}-\phi \alpha_{1} \beta_{1}+\zeta \beta_{1} \gamma_{1} \\
0
\end{array}\right)
\end{aligned}
$$

i.e.

$$
\begin{gathered}
\left(\begin{array}{cccc}
2 i \omega_{0}+\alpha+\lambda_{1} F^{*} & -\lambda & \lambda_{1} X^{*} & 0 \\
\theta N^{*} & 2 i \omega_{0}+\frac{s N^{*}}{L} & -\pi \phi N^{*} & 0 \\
0 & \phi F^{*} & 2 i \omega_{0}+\frac{u F^{*}}{M} & -\zeta F^{*} \\
0 & 0 & \gamma e^{-2 i \omega_{0} \tau_{k}} & 2 i \omega_{0}+\delta_{0}
\end{array}\right) \\
\times\left(\begin{array}{c}
F_{1}^{(1)} \\
F_{1}^{(2)} \\
F_{1}^{(3)} \\
F_{1}^{(4)}
\end{array}\right)=\left(\begin{array}{c}
-2 \lambda_{1} \beta_{1} \\
-2 \frac{s}{L} \alpha_{1}^{2}-2 \theta \alpha_{1}+2 \pi \phi \alpha_{1} \beta_{1} \\
-2 \frac{u}{M} \beta_{1}^{2}-2 \phi \alpha_{1} \beta_{1}+2 \zeta \beta_{1} \gamma_{1} \\
0
\end{array}\right)
\end{gathered}
$$

Similarly using Eqs. (60) and (64) in (62), we get 


$$
\begin{gathered}
\left(\begin{array}{cccc}
\alpha+\lambda_{1} F^{*} & -\lambda & \lambda_{1} X^{*} & 0 \\
\theta N^{*} & \frac{s N^{*}}{L} & -\pi \phi N^{*} & 0 \\
0 & \phi F^{*} & \frac{u F^{*}}{M} & -\zeta F^{*} \\
0 & 0 & \gamma & \delta_{0}
\end{array}\right)\left(\begin{array}{c}
F_{2}^{(1)} \\
F_{2}^{(2)} \\
F_{2}^{(3)} \\
F_{2}^{(4)}
\end{array}\right) \\
=\left(\begin{array}{c}
-2 \frac{s}{L}\left|\alpha_{1}\right|^{2}-2 \theta \operatorname{Re}\left\{\alpha_{1}\right\}+2 \pi \phi \operatorname{Re}\left\{\alpha_{1} \overline{\beta_{1}}\right\} \\
-2 \frac{u}{M}\left|\beta_{1}\right|^{2}-2 \phi \operatorname{Re}\left\{\alpha_{1} \overline{\beta_{1}}\right\}+2 \zeta \operatorname{Re}\left\{\beta_{1} \overline{\gamma_{1}}\right\} \\
0
\end{array}\right) .
\end{gathered}
$$

We solve the systems (66) and (67) for $F_{1}$ and $F_{2}$, respectively and using these values, we determine $W_{20}$ and $W_{11}$ and hence $g_{21}$. Now to determine the direction, stability and period of bifurcating periodic solutions of system (1) at the critical value $\tau=\tau_{k}$, we compute the following quantities:

$c_{1}(0)=\frac{i}{2 \omega_{0} \tau_{k}}\left(g_{11} g_{20}-2\left|g_{11}\right|^{2}-\frac{\left|g_{02}\right|^{2}}{3}\right)+\frac{g_{21}}{2}$,

$\mu_{2}=-\frac{\operatorname{Re}\left\{c_{1}(0)\right\}}{\operatorname{Re}\left\{\Psi^{\prime}\left(\tau_{k}\right)\right\}}$,

$\beta_{2}=2 \operatorname{Re}\left\{c_{1}(0)\right\}$,

$T_{2}=-\frac{\operatorname{Im}\left\{c_{1}(0)\right\}+\mu_{2} \operatorname{Im}\left\{\Psi^{\prime}\left(\tau_{k}\right)\right\}}{\omega_{0} \tau_{k}}$.

Now using the results of Hassard et al. (1981), we have the following theorem:

Theorem 5 If $\mu_{2}>0\left(\mu_{2}<0\right)$, then the Hopf-bifurcation is supercritical (subcritical) and the bifurcating periodic solutions exist for $\tau>\tau_{k}\left(\tau<\tau_{k}\right)$. The bifurcating periodic solution is stable (unstable) if $\beta_{2}<0 \quad\left(\beta_{2}>0\right)$ and the period increases (decreases) if $T_{2}>0\left(T_{2}<0\right)$.

\section{Numerical simulations}

\section{Parameter estimation}

The model parameters are estimated by using the time series data of atmospheric concentration of $\mathrm{CO}_{2}$, human population and forest biomass. The annual time series data for average atmospheric concentration of $\mathrm{CO}_{2}$ for the period 1961-2011 is taken from NOAA-ESRL data set (NOAA 2014). The data for world population for the period 1961-2011 is obtained from the United Nations
Population Division (UNPD 2012). The global forest biomass data for the years 1990, 2000, 2005 and 2010 is taken from FAO (2010). The average per capita $\mathrm{CO}_{2}$ emission for the period 1990-2011 is 4.5 metric tons per year (EDGAR 2014), which is equivalent to $0.576 \times 10^{-9} \mathrm{ppm}$ per person per year. Thus, the value of $\lambda$ is taken to be $0.576 \times$ $10^{-3} \mathrm{ppm}$ per million persons per year. Since the overall atmospheric life-time of $\mathrm{CO}_{2}$ is from 30 to 95 years (Jacobson 2005), $\alpha=1 / 62.5=0.016$ per year. Since in the period 1961-2011, reforestation efforts were not made at a significant level, while fitting the data, it is assumed that there is no reforestation effort in this period, i.e., $\zeta=0$, $\gamma=0, \delta_{0}=0$. In absence of appropriate data regarding other parameters, the model system (1) is calibrated for different values of parameters $Q_{0}, \lambda_{1}, s, L, u, M, \theta, \pi, \phi$ and find the best fit for $Q_{0}=5, \lambda_{1}=4.8 \times 10^{-9}, s=0.032$, $L=10,000, u=0.013, M=750,000, \theta=0.000001, \pi=$ 0.00004 and $\phi=0.71 \times 10^{-6}$.

Thus, we have the following set of estimated parameter values,

$$
\begin{gathered}
Q_{0}=5, \lambda=0.576 \times 10^{-3}, \alpha=0.016, \lambda_{1}=4.8 \times 10^{-9}, \\
s=0.032, L=10,000, u=0.013, M=750,000, \\
\theta=0.000001, \pi=0.00004, \phi=0.71 \times 10^{-6} .
\end{gathered}
$$

Since in 1961 the average atmospheric concentration of $\mathrm{CO}_{2}$ was 317.64 ppm (NOAA 2014) and world population was 3082.83 million (UNPD 2012), we take $X(0)=317.64$ ppm and $N(0)=3082.83$ million. In absence of data of forest biomass for the year 1961, it is taken that $F(0)=$ 640,000 million metric tons.

The value of R-squared for the actual and fitted data of atmospheric $\mathrm{CO}_{2}$, human population and forest biomass is calculated to be $0.9992,0.9996$ and 0.9962 , respectively. This shows the strong correlation between the actual data and the model projections. The actual data and the model projections of the variables $X, N$ and $F$ are plotted in Fig. 1 . It is evident from this figure that the concentration of $\mathrm{CO}_{2}$, human population and forest biomass as projected by our model are very near to the actual value. This ensures the validity of our model.

\section{Sensitivity analysis}

To assess the impact of changes in the parameters $\phi, \zeta$ and $\gamma$ on the behavior of (1), the basic sensitivity analysis of the model (1) for these parameters is performed following Bortz and Nelson (2004) and Misra and Verma (2014, 2015). The sensitivity systems with respect to parameters $\phi, \zeta$ and $\gamma$ are given by 


$$
\begin{aligned}
\dot{X}_{\phi}(t, \phi)= & \lambda N_{\phi}(t, \phi)-\alpha X_{\phi}(t, \phi)-\lambda_{1} X_{\phi}(t, \phi) F(t, \phi) \\
& -\lambda_{1} X(t, \phi) F_{\phi}(t, \phi), \\
\dot{N}_{\phi}(t, \phi)= & s\left(1-\frac{2 N(t, \phi)}{L}\right) N_{\phi}(t, \phi)-\theta X_{\phi}(t, \phi) N(t, \phi) \\
& -\theta X(t, \phi) N_{\phi}(t, \phi)+\pi \phi N_{\phi}(t, \phi) F(t, \phi) \\
& +\pi \phi N(t, \phi) F_{\phi}(t, \phi)+\pi N(t, \phi) F(t, \phi), \\
\dot{F}_{\phi}(t, \phi)= & u\left(1-\frac{2 F(t, \phi)}{M}\right) F_{\phi}(t, \phi)-N(t, \phi) F(t, \phi) \\
& -\phi N_{\phi}(t, \phi) F(t, \phi)-\phi N(t, \phi) F_{\phi}(t, \phi) \\
& +\zeta F_{\phi}(t, \phi) R(t, \phi)+\zeta F(t, \phi) R_{\phi}(t, \phi), \\
\dot{R}_{\phi}(t, \phi)= & -\gamma F_{\phi}(t-\tau, \phi)-\delta_{0} R_{\phi}(t, \phi), \\
\dot{X}_{\zeta}(t, \zeta)= & \lambda N_{\zeta}(t, \zeta)-\alpha X_{\zeta}(t, \zeta)-\lambda_{1} X_{\zeta}(t, \zeta) F(t, \zeta) \\
& -\lambda{ }_{1} X(t, \zeta) F_{\zeta}(t, \zeta), \\
\dot{N}_{\zeta}(t, \zeta)= & s\left(1-\frac{2 N(t, \zeta)}{L}\right) N_{\zeta}(t, \zeta)-\theta X_{\zeta}(t, \zeta) N(t, \zeta) \\
& -\theta X(t, \zeta) N_{\zeta}(t, \zeta)+\pi \phi N_{\zeta}(t, \zeta) F(t, \zeta) \\
& +\pi \phi N(t, \zeta) F_{\zeta}(t, \zeta), \\
\dot{F}_{\zeta}(t, \zeta)= & u\left(1-\frac{2 F(t, \zeta)}{M}\right) F_{\zeta}(t, \zeta)-\phi N_{\zeta}(t, \zeta) F(t, \zeta) \\
& -\phi N(t, \zeta) F_{\zeta}(t, \zeta)+\zeta F_{\zeta}(t, \zeta) R(t, \zeta) \\
& +\zeta F(t, \zeta) R_{\zeta}(t, \zeta)+F(t, \zeta) R(t, \zeta), \\
\dot{R}_{\zeta}(t, \zeta)= & -\gamma F_{\zeta}(t-\tau, \zeta)-\delta_{0} R_{\zeta}(t, \zeta),
\end{aligned}
$$

and

$$
\begin{aligned}
\dot{X}_{\gamma}(t, \gamma)= & \lambda N_{\gamma}(t, \gamma)-\alpha X_{\gamma}(t, \gamma)-\lambda_{1} X_{\gamma}(t, \gamma) F(t, \gamma) \\
& -\lambda_{1} X(t, \gamma) F_{\gamma}(t, \gamma) \\
\dot{N}_{\gamma}(t, \gamma)= & s\left(1-\frac{2 N(t, \gamma)}{L}\right) N_{\gamma}(t, \gamma)-\theta X_{\gamma}(t, \gamma) N(t, \gamma) \\
& -\theta X(t, \gamma) N_{\gamma}(t, \gamma)+\pi \phi N_{\gamma}(t, \gamma) F(t, \gamma) \\
& +\pi \phi N(t, \gamma) F_{\gamma}(t, \gamma) \\
\dot{F}_{\gamma}(t, \gamma)= & u\left(1-\frac{2 F(t, \gamma)}{M}\right) F_{\gamma}(t, \gamma)-\phi N_{\gamma}(t, \gamma) F(t, \gamma) \\
& -\phi N(t, \gamma) F_{\gamma}(t, \gamma)+\zeta F_{\gamma}(t, \gamma) R(t, \gamma) \\
& +\zeta F(t, \gamma) R_{\gamma}(t, \gamma), \\
\dot{R}_{\gamma}(t, \gamma)= & -\gamma F_{\gamma}(t-\tau, \gamma)+(M-F(t-\tau, \gamma))-\delta_{0} R_{\gamma}(t, \gamma),
\end{aligned}
$$

respectively. Here, $X_{\phi}(t, \phi)$ denotes the sensitivity function of $X$ with respect to parameter $\phi$, i.e., $X_{\phi}(t, \phi)=\frac{\partial}{\partial \phi} X(t, \phi)$.

To show the impact of doubling of parameters $\phi, \zeta$ and $\gamma$ on the state variables, semi-relative sensitivity solutions have been calculated for the best fitted data (72) along with $\zeta=0.0000026, \quad \gamma=0.0008, \quad \delta_{0}=0.0002 \quad$ and $\tau=5\left(<\tau_{0}\right)$. The semi-relative sensitivity solutions are depicted in Fig. 2. From this figure, it is clear that doubling of the parameter $\phi$ causes an increase of $7.5 \mathrm{ppm}$ in the
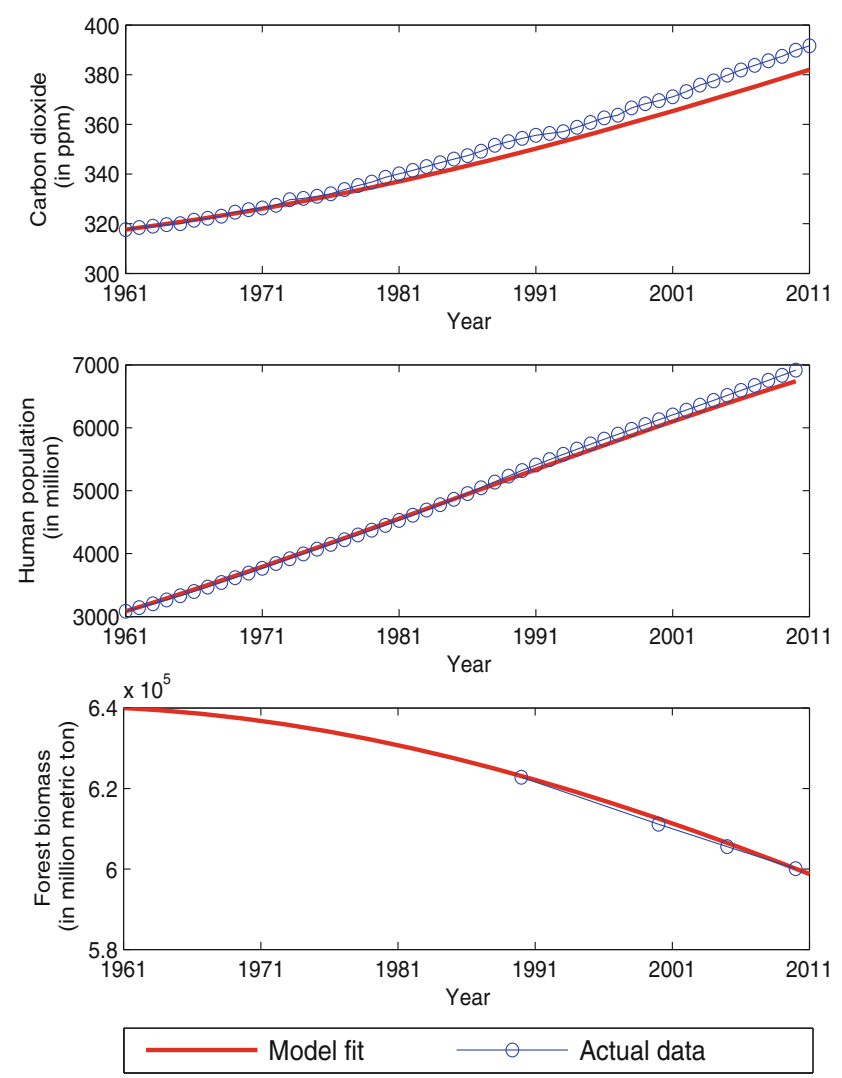

Fig. 1 Comparison between model fit and actual data of atmospheric concentration of carbon dioxide, human population and forest biomass

concentration of atmospheric $\mathrm{CO}_{2}$ over a period of 80 years. Doubling of parameters $\zeta$ and $\gamma$ cause drop of 15 and $14.4 \mathrm{ppm}$, respectively in the concentration of atmospheric $\mathrm{CO}_{2}$ over the period of 80 years. From the second and third plot of the Fig. 2, it can be noted that doubling of parameters $\zeta$ and $\gamma$ leads to increase in the human population and forest biomass. Doubling of parameter $\phi$ leads to increase in the human population and decrease in forest biomass. From this sensitivity analysis, it is clear that the parameters $\phi, \zeta$ and $\gamma$ have significant impact over the dynamics of the system.

\section{Validation of analytical results}

The numerical simulation performed by using MATLAB 7.5.0 for the fitted data (72) along with $\zeta=0.0000026$, $\gamma=0.0008, \delta_{0}=0.0002$ reveals that the conditions for feasibility of the coexistence equilibrium $E_{4}$ [i.e., (2) and (3)] are satisfied. The components of the interior equilibrium $E_{4}$ are obtained as: $X^{*}=544.2660 \mathrm{ppm}, N^{*}=$ 9836.5671 million, $F^{*}=7.493296 \times 10^{5}$ million metric tons, $R^{*}=2681.6700$ million dollars. In the absence of delay (i.e., $\tau=0$ ), the eigenvalues of the variational matrix 
Fig. 2 Semi-relative sensitivity solutions for the state variables with respect to parameters $\phi, \zeta$ and $\gamma$
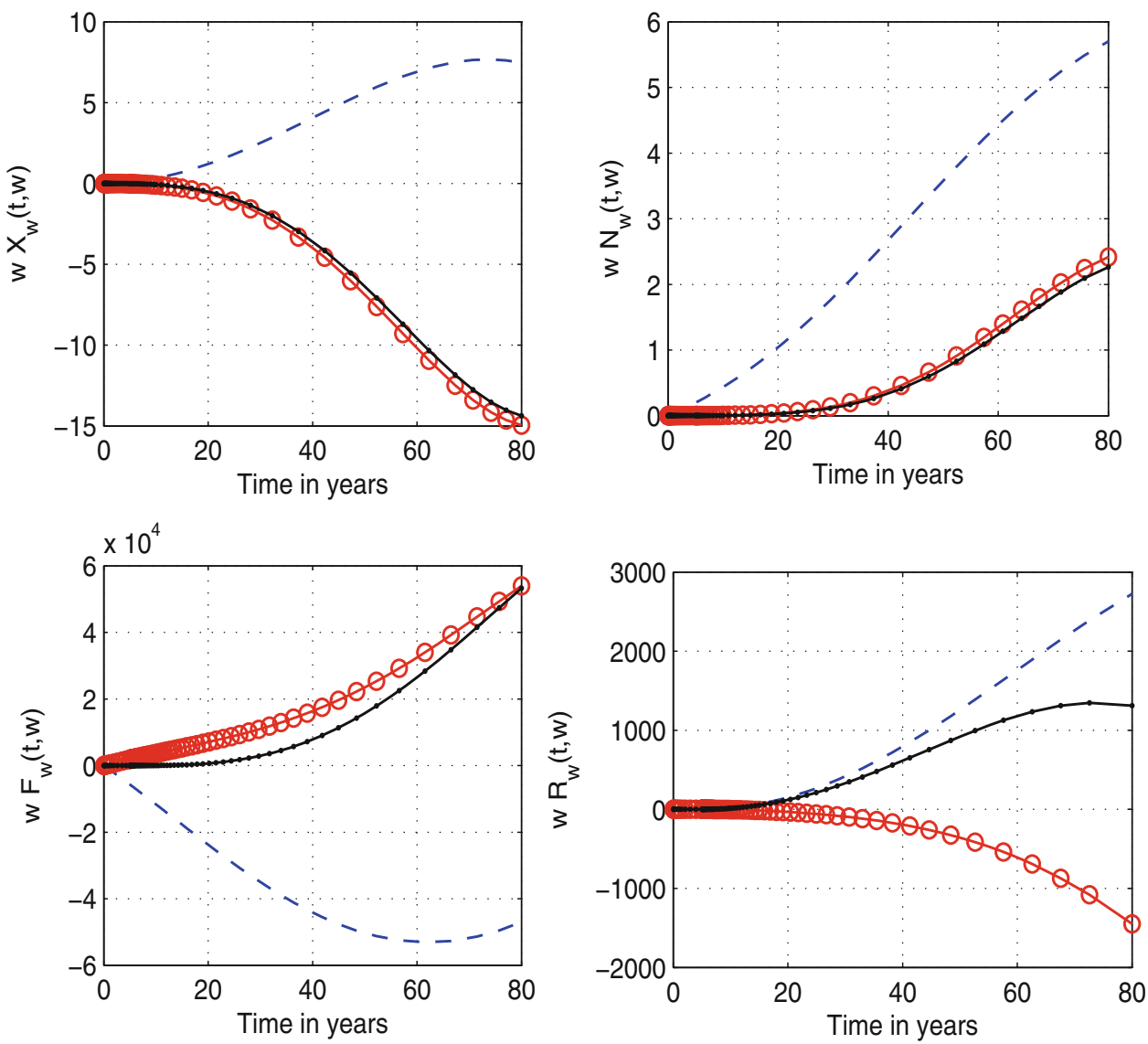

corresponding to equilibrium $E_{4}$ for the system (1) are $-0.0201, \quad-0.0310, \quad-0.0066+0.0390 i \quad$ and $-0.0066-0.0390 i$, all negative or with negative real part. Thus in the absence of delay the interior equilibrium $E_{4}$ is locally asymptotically stable. For the above set of parameter values, the condition (A4) for existence of a pair of purely imaginary roots of characteristic equation (15) is also satisfied. The numerical value of $\tau_{0}$ using Eq. (20) is found to be 8.6178 years. The values of $\mu_{2}, \beta_{2}$ and $T_{2}$ are calculated as $\mu_{2}=0.000631, \beta_{2}=-8.82299 \times 10^{-7}$ and $T_{2}=0.8505 \times 10^{-4}$. Since $\mu_{2}>0, \beta_{2}<0$ and $T_{2}>0$, it follows from Theorem 5 that the Hopf-bifurcation is supercritical, the bifurcating periodic solutions are stable and their period increases. The variation in variables $X, N$, $F$ and $R$ for $\tau=4$ years $\left(\tau<\tau_{0}\right)$ and $\tau=11$ years $\left(\tau>\tau_{0}\right)$ have been drawn in Fig. 3. From this figure, it can be noted that for $\tau=4$ years, all the variables approach their equilibrium values, but for $\tau=11$ years, all the variables show oscillatory behavior. This shows that interior equilibrium $E_{4}$ is stable for $\tau<\tau_{0}$, but when the time delay exceeds the critical value $\tau_{0}$, the stable solution becomes periodic.
The bifurcation diagrams of atmospheric concentration of $\mathrm{CO}_{2}$ and forest biomass with respect to $\tau$ are shown in Fig. 4. This figure shows the change in dynamics of atmospheric concentration of $\mathrm{CO}_{2}$ and forest biomass as the parameter $\tau$ varies. From this figure, we can see that for small values of time delay $\tau$, atmospheric concentration of $\mathrm{CO}_{2}$ and forest biomass stabilize to their equilibrium values, but as the value of time delay $\tau$ crosses the critical value $\tau_{0}(=8.6$ years $)$, the system loses its stability and undergoes Hopf-bifurcation. This implies that if the reforestation efforts are applied on the basis of the measurements of forest biomass which are taken more than $\tau_{0}$ years ago, then levels of atmospheric $\mathrm{CO}_{2}$ and forest biomass do not settle down to the equilibrium values.

\section{Discussion and recommendations}

Deforestation is one of the root cause behind the enhanced concentration of atmospheric carbon dioxide and the associated climate changes. Reforestation is an avenue to 
Fig. 3 Variation of $X, N, F$ and $R$ with respect to time $t$ for $\tau=$ 4 years (dashed line) and $\tau=11$ years (solid line)
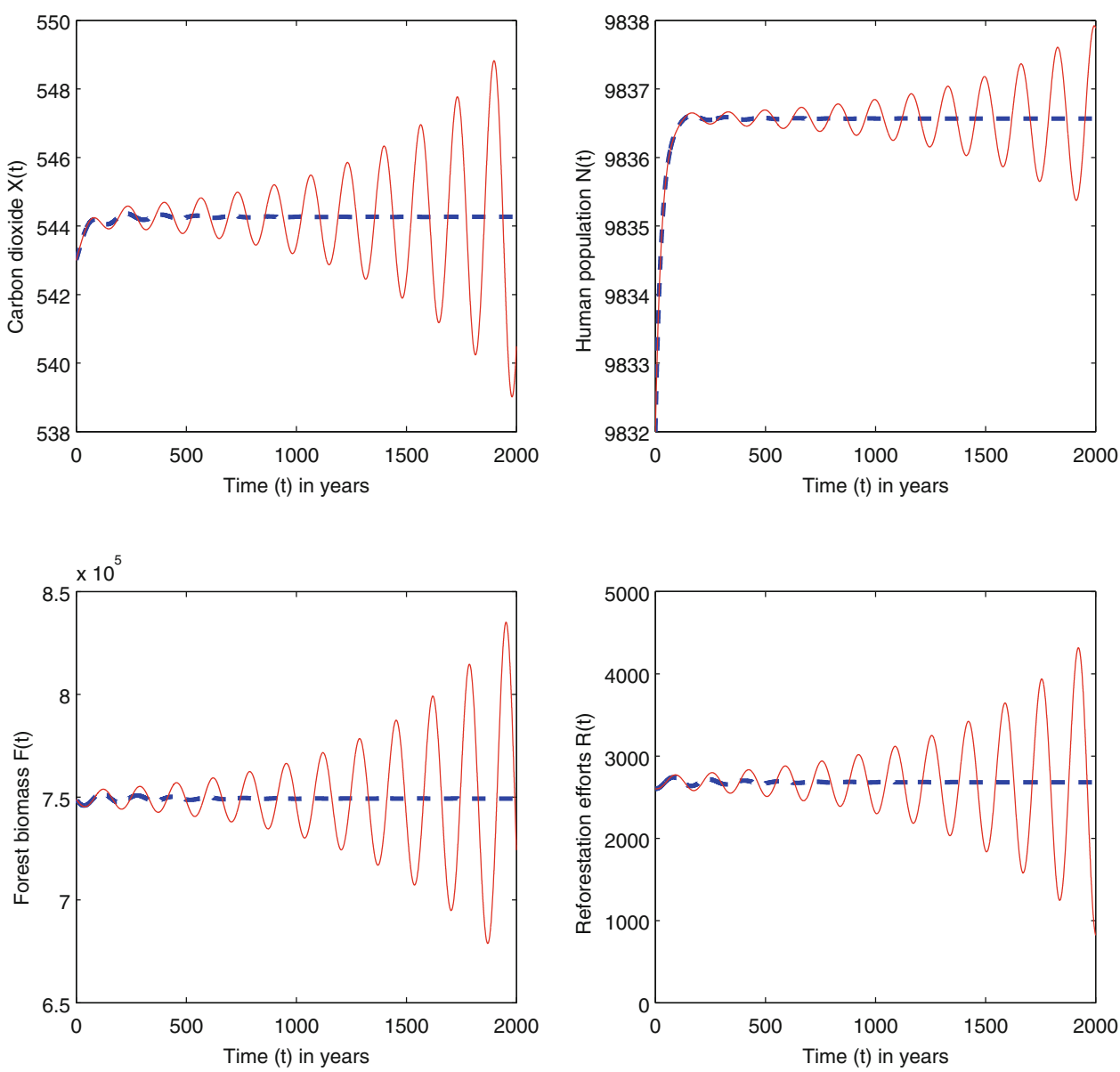

reduce the atmospheric concentration of carbon dioxide. But the reforestation programs are usually developed on the basis of the measurements of forest biomass that have been taken some time earlier. In this paper, a nonlinear mathematical model is proposed and analyzed to study the effects of reforestation and the delay involved in between the measurement of forest data and implementation of reforestation efforts on the control of atmospheric level of $\mathrm{CO}_{2}$. We have assumed that the reforestation efforts are implemented with a rate proportional to the difference of carrying capacity of forest biomass and its value measured $\tau$ times earlier. The model analysis shows that the concentration of atmospheric $\mathrm{CO}_{2}$ decreases as reforestation efforts increase. The conditions for local as well as global stability of interior equilibrium $E_{4}$ have been obtained in absence of time delay. To investigate the impact of increase in time delay over the stability of equilibrium $E_{4}$, the local stability of interior equilibrium $E_{4}$ is analyzed in presence of delay. It is found that the stability of the equilibrium $E_{4}$ is preserved for all time delay provided the conditions stated in Theorem 3 are satisfied. Moreover, if these conditions are not satisfied, then model analysis provides the conditions under which the equilibrium $E_{4}$ loses its stability as time delay $\tau$ crosses some critical value $\tau_{0}$ and a family of periodic solutions arises through Hopfbifurcation. This critical value has been obtained analytically and is given by Eq. (20). The direction of Hopfbifurcation and stability of bifurcating periodic solutions are also investigated by using the center manifold theorem and normal form theory.

Sensitivity analysis shows that deforestation rate coefficient $\phi$, implementation rate coefficient of reforestation efforts $\gamma$ and efficiency of reforestation efforts to increase forest biomass $\zeta$ have significant impact on the dynamics of the system. Through increasing $\gamma$ and $\zeta$, the atmospheric level of $\mathrm{CO}_{2}$ can be significantly reduced. Thus, reforestation efforts have the potential to control the atmospheric concentration of $\mathrm{CO}_{2}$ but longer delays between the measurement of forest data and implementation of reforestation efforts may have destabilizing effect over the dynamics of the system. The numerical simulations clearly show the effect of increase in time delay over the dynamics of the system. It is shown that when time delay exceeds a critical value, the interior equilibrium loses its stability and periodic solution arises via Hopf-bifurcation. The analytical expression for this critical value of time delay is derived and is given by Eq. (20). The critical value of time delay is crucial to be determined for development of 
Fig. 4 Bifurcation diagrams of atmospheric $\mathrm{CO}_{2}$ and forest biomass with respect to $\tau$
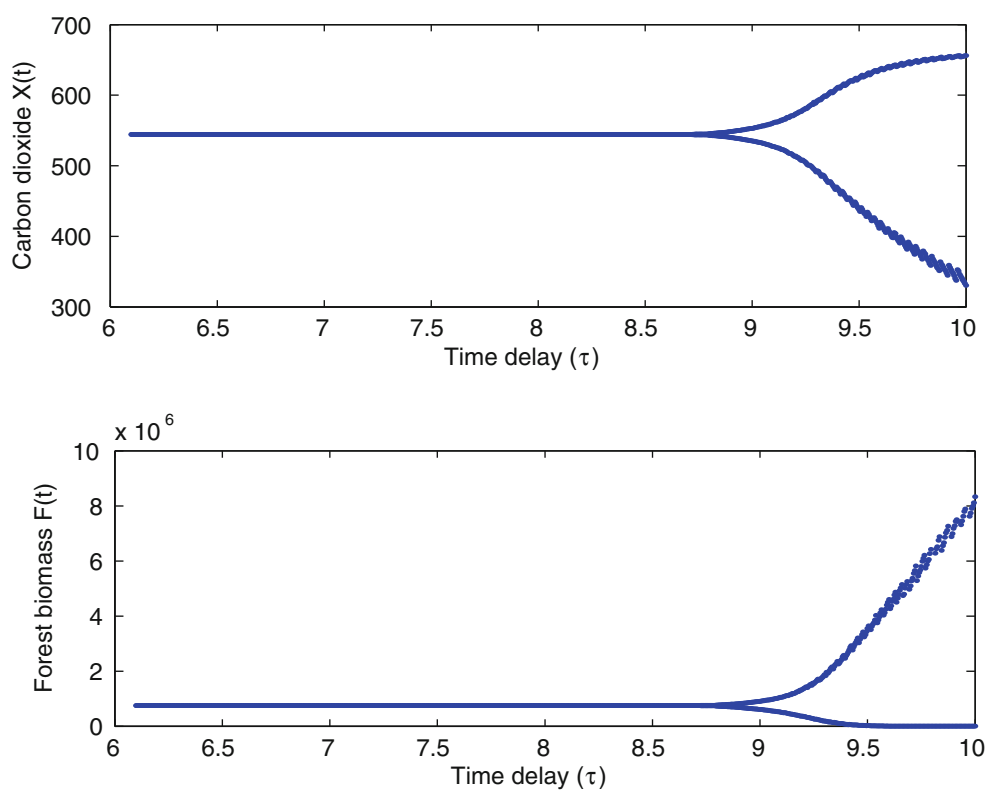
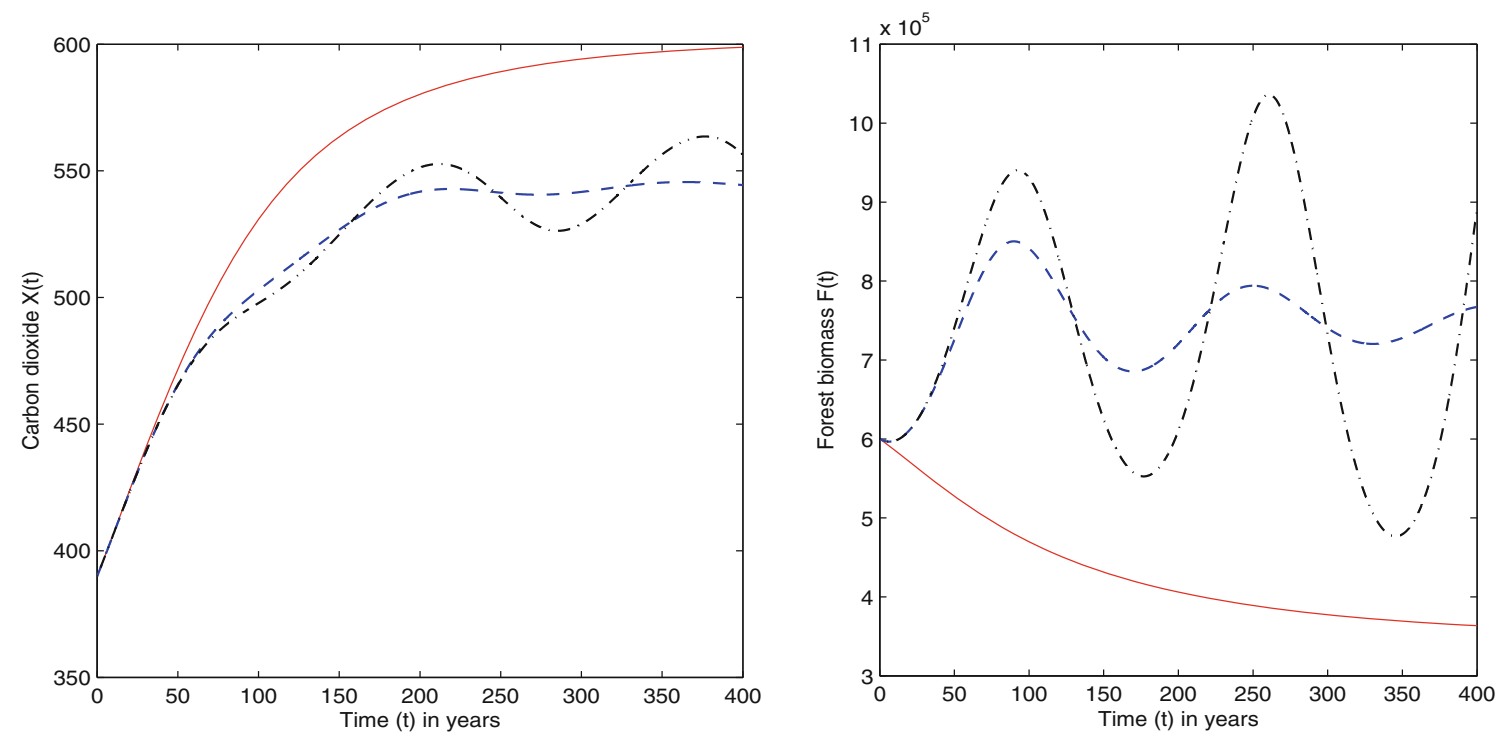

Without reforestation efforts $\quad-\quad-$ With reforestation efforts $(\tau=2) \quad-\quad \cdot-$ With reforestation efforts $(\tau=12)$

Fig. 5 Future variations in atmospheric concentration of $\mathrm{CO}_{2}$ and forest biomass in presence and absence of reforestation efforts

potential reforestation policies. For if the available data of forest biomass is older than this critical limit, the levels of atmospheric $\mathrm{CO}_{2}$ and forest biomass will fluctuate largely. Consequently, reduction and stabilization of atmospheric concentration of $\mathrm{CO}_{2}$ via reforestation efforts will become a difficult task. Sensitivity analysis shows that increase in deforestation rate significantly increase the atmospheric concentration of $\mathrm{CO}_{2}$. Also, from the condition of global stability of the equilibrium $E_{4}$ [i.e., condition (10)], it can be seen that the deforestation rate coefficient ' $\phi$ ' has destabilizing effect over the dynamics of the system. Thus, apart from reforestation, policies should also focus to control the accelerating deforestation rates in various parts of the world. However, due to rapid growth in population and industrialization, use of forest biomass can be reduced only upto a certain level. In this scenario, reforestation is the key to control the $\mathrm{CO}_{2}$ emissions due to deforestation. But a potential reforestation policy can be developed only 
if it is based on the recent data of forest biomass. Thus government should also focus on more frequent and accurate estimation of forest biomass, so that better reforestation programmes can be developed.

To depict the effect of implementation of reforestation efforts and the delay involved in their implementation over the future levels of atmospheric $\mathrm{CO}_{2}$ and forest biomass, the variations in these two variables with respect to time in presence and absence of reforestation efforts are shown in Fig. 5. The initial time is taken as the year 2010 and the initial conditions are $X(0)=389.84 \mathrm{ppm}, \quad N(0)=$ 6834.722 million, $F(0)=600,066$ million metric tons and $R(0)=200$ million dollars [since in 2010, the average atmospheric concentration of $\mathrm{CO}_{2}$ was $389.84 \mathrm{ppm}$ (NOAA 2014), the world population was 6916.183 million (UNPD 2012) and the forest biomass was 600066 million metric tons (FAO 2010)]. From this figure, it may be noted that when reforestation efforts are not applied (i.e., $\zeta=0$, $\left.\gamma=0, \delta_{0}=0\right)$, the forest biomass decreases with time and the atmospheric concentration of $\mathrm{CO}_{2}$ settles down to a level of $600 \mathrm{ppm}$. But, when reforestation efforts (with $\zeta=0.0000026, \gamma=0.0008, \delta_{0}=0.0002$ and $\tau=2$ years) are applied, forest biomass increases and atmospheric concentration of $\mathrm{CO}_{2}$ settles down to a comparatively low level $(544.5 \mathrm{ppm})$. This shows that the reforestation efforts may be used to control the atmospheric concentration of $\mathrm{CO}_{2}$. But, if reforestation efforts are applied with longer time delay ( $\tau=12$ years), then levels of atmospheric carbon dioxide and forest biomass fluctuate largely and do not get stabilized. Thus, as already stated, for the development of potential reforestation polices, the data of the forest biomass used should not be too much old.

Acknowledgements Authors are thankful to the handling editor and reviewer for their useful suggestions those improved the quality of the paper. The first author thankfully acknowledges the University Grants Commission, New Delhi, India for providing financial support under major research project (MRP-MAJOR-MATH-2013-26774). The second author is thankful to National Board of Higher Mathematics, Department of Atomic Energy, Government of India for providing financial support in form of postdoctoral fellowship (No:2/ 40(7)/2015/R\&D-II/4951).

\section{References}

Bortz DM, Nelson PW (2004) Sensitivity analysis of a nonlinear lumped parameter model of HIV infection dynamics. Bull Math Biol 66:1009-1026

Brown S (1993) Tropical forests and the global carbon cycle: the need for sustainable land-use patterns. Agric Ecosyst Environ 46:31-44

Caetano MAL, Gherardi DFM, Yoneyama T (2011) An optimized policy for the reduction of $\mathrm{CO}_{2}$ emission in the Brazilian Legal Amazon. Ecol Model 222:2835-2840

Casper JK (2010) Greenhouse gases: worldwide impacts. Facts On File Inc., New York
Dubey B, Sharma S, Sinha P, Shukla J (2009) Modelling the depletion of forestry resources by population and population pressure augmented industrialization. Appl Math Model 33:3002-3014

EDGAR (2014) $\mathrm{CO}_{2}$ time series 1990-2011 per capita for world countries. http://edgar.jrc.ec.europa.eu/overview.php?v=CO2ts_ pc1990-2011. Accessed 20 Apr 2015

FAO (2010) Global forest resources assessment 2010: main report, FAO forestry paper no. 163. ISBN:978-92-5-106654-6

Freedman HI, Rao VSH (1983) The trade-off between mutual interference and time lags in predator-prey systems. Bull Math Biol 45:991-1004

Gopalsamy K (1992) Stability and oscillations in delay differential equations of population dynamics. Kluwer Academic Publishers, Dordrecht, Norwell

Goreau TJ (1992) Control of atmospheric carbon dioxide. Glob Environ Chang 2:5-11

Hartwick JM (2005) Deforestation and population increase. In: Kant S, Berry RA (eds) Institutions, sustainability, and natural resources: institutions for sustainable forest management. Springer, Netherlands, pp 155-191

Hassard BD, Kazarinoff ND, Wan YH (1981) Theory and applications of Hopf-bifurcation. Cambridge University Press, Cambridge

Houghton RA (2005) Aboveground forest biomass and the global carbon balance. Glob Change Biol 11:945-958

IPCC (2001) The carbon cycle and atmospheric carbon dioxide. In: Houghton JT, Ding Y, Griggs DJ, Noguer M, Van der Linden PJ, Dai X, Maskell K, Johnson CA (eds) Climate change 2001: the scientifc basis. Contribution of working group I to the third assessment report of the intergovernmental panel on climate change. Cambridge University Press, Cambridge. New York

IPCC (2007a) Summary for policymakers. In: Solomon S, Qin D, Manning M, Chen Z, Marquis M, Averyt KB, Tignor M. Miller HL (eds) Climate change 2007: the physical science basis. Contribution of working group I to the fourth assessment report of the intergovernmental panel on climate change. Cambridge University Press, Cambridge, New York

IPCC (2007b) Technical summary. In: Solomon S, Qin D, Manning M, Chen Z, Marquis M, Averyt KB, Tignor M, Miller HL (eds) Climate change 2007: the physical science basis. Contribution of working group I to the fourth assessment report of the intergovernmental panel on climate change. Cambridge University Press, Cambridge, New York

Jacobson MZ (2005) Correction to Control of fossil-fuel particulate black carbon and organic matter, possibly the most effective method of slowing global warming. J Geophys Res 110:D14105(1-5)

McMichael AJ, Woodruff RE, Hales S (2006) Climate change and human health: present and future risks. Lancet 367:859-869

Misra AK, Verma M (2013) A mathematical model to study the dynamics of carbon dioxide gas in the atmosphere. Appl Math Comput 219:8595-8609

Misra AK, Verma M (2014) Modeling the impact of mitigation options on methane abatement from rice fields. Mitig Adapt Strateg Glob Change 19:927-945

Misra AK, Verma M (2015) Impact of environmental education on mitigation of carbon dioxide emissions: a modelling study. Int $\mathbf{J}$ Glob Warm 7:466-486

Newell ND, Marcus L (1987) Carbon dioxide and people. Palaios 2:101-103

Nikol'skii MS (2010) A controlled model of carbon circulation between the atmosphere and the ocean. Comput Math Model 21:414-424

NOAA (2014) Atmospheric $\mathrm{CO}_{2}$ concentrations (ppm) since March 1958. http://co2now.org/Current-CO2/CO2-Now/noaa-maunaloa-co2-data.html. Accessed 20 Apr 2015 
Onozaki K (2009) Population is a critical factor for global carbon dioxide increase. J Health Sci 55:125-127

Shukla JB, Dubey B (1997) Modelling the depletion and conservation of forestry resources: effects of population and pollution. J Math Biol 36:71-94

Shukla JB, Chauhan MS, Sundar S, Naresh R (2015) Removal of carbon dioxide from the atmosphere to reduce global warming: a modeling study. Int J Glob Warm Gases 7:270-292

Tennakone K (1990) Stability of the biomass-carbon dioxide equilibrium in the atmosphere: mathematical model. Appl Math Comput 35:125-130

UNPD (2012) United nations population division, department of economic and social affairs, World population prospects: the
2012 revision, total population both sexes. http://esa.un.org/wpp/ Excel-Data/population.htm. Accessed 20 Apr 2015

WHO (2009) Global health risks: mortality and burden of disease attributable to selected major risks. http://www.who.int/ healthinfo/lobal_burden_disease/GlobalHealthRisks_report_full. pdf. Accessed 15 Apr 2015. ISBN: 978-92-4-156387-1

Woodwell GM, Hobbie JE, Houghton RA, Melillo JM, Moore B, Peterson BJ, Shaver GR (1983) Global deforestation: contribution to atmospheric carbon dioxide. Science 222:1081-1086 